\title{
NULL CONTROLLABILITY OF THE HEAT EQUATION WITH BOUNDARY FOURIER CONDITIONS: THE LINEAR CASE*
}

\author{
Enrique Fernández-CarA ${ }^{1}$, Manuel González-Burgos ${ }^{1}$, Sergio Guerrero ${ }^{2}$ And \\ JEAN-PIERRE PUEL ${ }^{3}$
}

\begin{abstract}
In this paper, we prove the global null controllability of the linear heat equation completed with linear Fourier boundary conditions of the form $\frac{\partial y}{\partial n}+\beta y=0$. We consider distributed controls with support in a small set and nonregular coefficients $\beta=\beta(x, t)$. For the proof of null controllability, a crucial tool will be a new Carleman estimate for the weak solutions of the classical heat equation with nonhomogeneous Neumann boundary conditions.
\end{abstract}

Mathematics Subject Classification. 35K20, 93B05.

Received February 17, 2005. Revised April 2, 2005.

\section{INTRODUCTION}

Let $\Omega \subset \mathbb{R}^{N}$ be a bounded connected open set whose boundary $\partial \Omega$ is regular enough $(N \geq 1)$. Let $\omega \subset \Omega$ be a (small) nonempty open subset and let $T>0$. We will use the notation $Q=\Omega \times(0, T)$ and $\Sigma=\partial \Omega \times(0, T)$ and we will denote by $n(x)$ the outward unit normal to $\Omega$ at $x \in \partial \Omega$. On the other hand, we will denote by $C$, $C_{1}, C_{2}, \ldots$ generic positive constants (usually depending on $\Omega$ and $\omega$ ).

We will consider the linear heat equation with linear Fourier (or Robin) conditions

$$
\begin{cases}y_{t}-\Delta y+B(x, t) \cdot \nabla y+a(x, t) y=v(x, t) 1_{\omega} & \text { in } Q, \\ \frac{\partial y}{\partial n}+\beta(x, t) y=0 & \text { on } \Sigma, \\ y(x, 0)=y^{0}(x) & \text { in } \Omega .\end{cases}
$$

Here, it will be assumed that the coefficients $a, B$ and $\beta$ satisfy

$$
a \in L^{\infty}(Q), \quad B \in L^{\infty}(Q)^{N}, \quad \beta \in L^{\infty}(\Sigma) .
$$

\footnotetext{
Keywords and phrases. Controllability, heat equation, Fourier conditions.

* This work has been partially supported by D.G.E.S. (Spain), Grant and BFM2003-06446.

${ }^{1}$ Dpto. E.D.A.N., Universidad de Sevilla, Aptdo. 1160, 41080 Sevilla, Spain; cara@us.es; manoloburgos@us.es; sguerrero@us.es

2 Laboratoire Jacques-Louis Lions, Université Pierre et Marie Curie, boîte courrier 187, 75035 Cedex 05, Paris, France; guerrero@ann.jussieu.fr

3 Laboratoire de Mathématiques Appliquées, Université de Versailles, St. Quentin, 45 avenue des États-Unis, 78035 Versailles, France; jppuel@cmapx.polytechnique.fr 
On the other hand, we suppose that $v \in L^{2}(\omega \times(0, T)), 1_{\omega}$ is the characteristic function of $\omega$ and $y^{0} \in L^{2}(\Omega)$. In $(1), y=y(x, t)$ is the state and $v=v(x, t)$ is the control. It is assumed that we can act on the system only through $\omega \times(0, T)$.

An illustrative interpretation of the data and variables in (1) is the following. The function $y$ can be viewed as the relative temperature of a body (with respect to the exterior surrounding air). The parabolic equation in (1) means, among other things, that a heat source $v 1_{\omega}$ acts on a part of the body. On the boundary, $-\frac{\partial y}{\partial n}$ must be viewed as the normal heat flux, directed inwards, up to a positive coefficient. Thus, the equality

$$
-\frac{\partial y}{\partial n}=\beta y
$$

means that this flux is a linear function of the temperature. Thus, it is reasonable to suppose that $\beta \geq 0$ (although this assumption will not be imposed in this paper).

The main goal of this paper is to analyze the controllability properties of (1). It will be said that this system is null controllable at time $T$ if, for each $y^{0} \in L^{2}(\Omega)$, there exists $v \in L^{2}(\omega \times(0, T))$ such that the associated solution satisfies

$$
y(x, T)=0 \quad \text { in } \Omega .
$$

The null controllability of linear parabolic equations has been intensively studied these last years; see for instance $[1,4,5,7,8]$.

In this paper, we will be concerned with (1), where the main difficulties arise from the particular form of the boundary condition. Indeed, it has been shown in $[2,5]$ that this is more difficult to analyze than the case of Dirichlet boundary conditions, considered in $[4,5,7]$.

More precisely, what has been proved until now is that (1) is null controllable with $B \equiv 0$ under the assumptions (2) whenever $\beta_{t} \in L^{\infty}(\Sigma)$. This was shown in [5]. However, it would be important to prove the null controllability of (1) without this regularity hypothesis on $\beta_{t}$ in view of applications to control systems with nonlinear boundary conditions.

The first main result in this paper concerns a Carleman inequality for a general (adjoint) system of the form

$$
\begin{cases}-\varphi_{t}-\Delta \varphi=f_{1}(x, t)+\nabla \cdot f_{2}(x, t) & \text { in } Q, \\ \left(\nabla \varphi+f_{2}(x, t)\right) \cdot n=f_{3}(x, t) & \text { on } \Sigma, \\ \varphi(x, T)=\varphi^{0}(x) & \text { in } \Omega,\end{cases}
$$

where $f_{1} \in L^{2}(Q), f_{2} \in L^{2}(Q)^{N}$ and $f_{3} \in L^{2}(\Sigma)$. Observe that, as long as $\varphi \in L^{2}(Q), \nabla \varphi+f_{2} \in L^{2}(Q)^{N}$ and $\nabla$. $\left(\nabla \varphi+f_{2}\right) \in H^{-1}\left(0, T ; L^{2}(\Omega)\right)$, we can give a sense to the boundary condition in the space $H^{-1}\left(0, T ; H^{-1 / 2}(\partial \Omega)\right)$.

We present now this result:

Theorem 1. Under the previous assumptions on $f_{1}, f_{2}$ and $f_{3}$, there exist $\bar{\lambda}, \sigma_{1}, \sigma_{2}$ and $C$, only depending on $\Omega$ and $\omega$, such that, for any $\lambda \geq \bar{\lambda}$, any $s \geq \bar{s}=\sigma_{1}\left(\mathrm{e}^{\sigma_{2} \lambda} T+T^{2}\right)$ and any $\varphi^{0} \in L^{2}(\Omega)$, the weak solution to (4) satisfies

$$
\begin{aligned}
& \iint_{Q} \mathrm{e}^{-2 s \alpha}\left(s \lambda^{2} \xi|\nabla \varphi|^{2}+s^{3} \lambda^{4} \xi^{3}|\varphi|^{2}\right) \mathrm{d} x \mathrm{~d} t+s^{2} \lambda^{3} \iint_{\Sigma} \mathrm{e}^{-2 s \alpha} \xi^{2}|\varphi|^{2} \mathrm{~d} \sigma \mathrm{d} t \\
& \leq C\left(\iint_{Q} \mathrm{e}^{-2 s \alpha}\left(\left|f_{1}\right|^{2}+s^{2} \lambda^{2} \xi^{2}\left|f_{2}\right|^{2}\right) \mathrm{d} x \mathrm{~d} t\right. \\
& \left.\quad+s \lambda \iint_{\Sigma} \mathrm{e}^{-2 s \alpha} \xi\left|f_{3}\right|^{2} \mathrm{~d} \sigma \mathrm{d} t+s^{3} \lambda^{4} \iint_{\omega \times(0, T)} \mathrm{e}^{-2 s \alpha} \xi^{3}|\varphi|^{2} \mathrm{~d} x \mathrm{~d} t\right) .
\end{aligned}
$$

Here, $\alpha=\alpha(x, t)$ and $\xi=\xi(x, t)$ are appropriate positive functions, again only depending on $\Omega$ and $\omega$. They are given below; see (13)-(14). 
As a consequence of Theorem 1, we can deduce an observability inequality for the adjoint system associated to (1). More precisely, let us consider the backward in time system

$$
\begin{cases}-\varphi_{t}-\Delta \varphi-\nabla \cdot(\varphi B(x, t))+a(x, t) \varphi=0 & \text { in } Q, \\ (\nabla \varphi+\varphi B(x, t)) \cdot n+\beta(x, t) \varphi=0 & \text { on } \Sigma, \\ \varphi(x, T)=\varphi^{0}(x) & \text { in } \Omega,\end{cases}
$$

where $\varphi^{0} \in L^{2}(\Omega)$. It will be seen that, for some $K$ of the form

$$
K=\mathrm{e}^{C\left(1+\frac{1}{T}+\|a\|_{\infty}^{2 / 3}+\|B\|_{\infty}^{2}+\|\beta\|_{\infty}^{2}\right)},
$$

the solutions of (6) satisfy

$$
\iint_{\Omega \times(T / 4,3 T / 4)}|\varphi|^{2} \mathrm{~d} x \mathrm{~d} t \leq K \iint_{\omega \times(0, T)}|\varphi|^{2} \mathrm{~d} x \mathrm{~d} t .
$$

Remark 1. In fact, (8) is not the unique way of saying that (6) is observable. It is indeed more frequent to use other inequalities of the form

$$
\|\varphi(\cdot, 0)\|_{L^{2}(\Omega)}^{2} \leq C \iint_{\omega \times(0, T)}|\varphi|^{2} \mathrm{~d} x \mathrm{~d} t
$$

for some $C$. The estimates (9) can be easily deduced from (8) and the energy inequalities satisfied by $\varphi$.

The second main result in this paper concerns the null controllability of (1). It is the following:

Theorem 2. Let us assume that (2) is satisfied. Then, for each $T>0,(1)$ is null controllable at time $T$ with controls $v \in L^{2}(\omega \times(0, T))$. Moreover, one can find $v$ such that

$$
\|v\|_{L^{2}(\omega \times(0, T))} \leq H\left\|y^{0}\right\|_{L^{2}},
$$

with a constant $H$ of the form

$$
H=\mathrm{e}^{C\left(1+\frac{1}{T}+\|a\|_{\infty}^{2 / 3}+\|B\|_{\infty}^{2}+\|\beta\|_{\infty}^{2}+T\left(\|a\|_{\infty}+\|B\|_{\infty}^{2}+\|\beta\|_{\infty}^{2}\right)\right)}
$$

for some $C=C(\Omega, \omega)$.

In the proof of Theorem 2, the main tool is the estimate (8). This arises from a general principle that asserts that the null controllability of (1) with controls in $L^{2}(\omega \times(0, T))$ (depending continuously on the data) is equivalent to the observability of (6). More details will be given below.

In a second part of this work, which will appear in a forthcoming paper, we will consider controllability questions for semilinear heat equations completed with nonlinear Fourier boundary conditions of the form

$$
\frac{\partial y}{\partial n}+f(y)=0 \quad \text { on } \quad \Sigma
$$

where $f: \mathbb{R} \mapsto \mathbb{R}$ is locally Lipschitz-continuous. For the analysis of these systems, Theorems 1 and 2 of the present paper will be crucial.

The rest of this paper is organized as follows. Section 2 is devoted to the proof of Theorem 1 . In Section 3 , we deduce the observability inequality (8) and we prove Theorem 2. For completeness, we have included an Appendix, where we give a detailed proof of the standard Carleman estimate for the solutions of the heat equation with homogeneous Neumann boundary conditions. (this estimate was already proved in [5]; however, in this paper, a careful study of the dependence of the constants on $s, \lambda$ and $T$ is needed). 


\section{Proof of Theorem 1}

The main arguments used below are similar to those in [6]. This is related to a general strategy which is used to relax the regularity assumptions on the various coefficients involved in the problem. Here, it will allow us to proceed without any kind of regularity on the coefficient $\beta=\beta(x, t)$.

Let us recall the definition of a weak solution: we say that $\varphi$ is a weak solution to (4) if it satisfies

$$
\left\{\begin{array}{l}
\varphi \in L^{2}\left(0, T ; H^{1}(\Omega)\right) \cap C^{0}\left([0, T] ; L^{2}(\Omega)\right), \\
-\left\langle\varphi_{t}, v\right\rangle_{\left(H^{1}(\Omega)\right)^{\prime}, H^{1}(\Omega)}+\int_{\Omega} \nabla \varphi \cdot \nabla v \mathrm{~d} x=\int_{\Omega} f_{1}(x, t) v \mathrm{~d} x \\
\quad-\int_{\Omega} f_{2}(x, t) \cdot \nabla v \mathrm{~d} x+\int_{\partial \Omega} f_{3}(x, t) v \mathrm{~d} \sigma \\
\text { a.e. in }(0, T), \quad \forall v \in H^{1}(\Omega), \\
\varphi(x, T)=\varphi^{0}(x) \text { in } \Omega .
\end{array}\right.
$$

It is well known that, for $f_{1} \in L^{2}(Q), f_{2} \in L^{2}(Q)^{N}, f_{3} \in L^{2}(\Sigma)$ and $\varphi^{0} \in L^{2}(\Omega)$, (4) possesses exactly one weak solution $\varphi$.

To prove the Carleman inequality (5), we will need two weight functions:

$$
\xi(x, t)=\frac{\mathrm{e}^{\lambda \eta^{0}(x)}}{t(T-t)}, \quad \alpha(x, t)=\frac{\mathrm{e}^{2 \lambda\left\|\eta^{0}\right\|_{\infty}}-\mathrm{e}^{\lambda \eta^{0}(x)}}{t(T-t)} .
$$

Here, $\lambda \geq 1$ is a parameter to be chosen below and $\eta^{0}=\eta^{0}(x)$ is a function satisfying

$$
\begin{aligned}
\eta^{0} \in C^{2}(\bar{\Omega}), & \eta^{0}(x)>0 \text { in } \Omega, \quad \eta^{0}(x)=0 \text { on } \partial \Omega, \\
& \left|\nabla \eta^{0}(x)\right|>0 \text { in } \overline{\Omega \backslash \omega^{\prime}},
\end{aligned}
$$

where $\omega^{\prime} \subset \subset \omega$ is a nonempty open set. The existence of $\eta^{0}$ satisfying (14) is proved in [5].

For the proof of Theorem 1, we will need an auxiliary result: a Carleman inequality for the solutions to the heat equation with homogeneous Neumann boundary conditions. This is given in the following result:

Lemma 1. Let $f \in L^{2}(Q)$ be given. There exist $\lambda^{*}, \sigma^{*}$ and $C$ only depending on $\Omega$ and $\omega$ such that, for any $\lambda \geq \lambda^{*}$, any $s \geq s^{*}(\lambda)=\sigma^{*}\left(\mathrm{e}^{2 \lambda\left\|\eta^{0}\right\|_{\infty}} T+T^{2}\right)$ and any $q^{0} \in L^{2}(\Omega)$, the weak solution to

$$
\begin{cases}-q_{t}-\Delta q=f(x, t) & \text { in } Q \\ \frac{\partial q}{\partial n}=0 & \text { on } \Sigma \\ q(x, T)=q^{0}(x) & \text { in } \Omega\end{cases}
$$

satisfies

$$
I_{s, \lambda}(q) \leq C\left(\iint_{Q} \mathrm{e}^{-2 s \alpha}|f|^{2} \mathrm{~d} x \mathrm{~d} t+s^{3} \lambda^{4} \iint_{\omega \times(0, T)} \mathrm{e}^{-2 s \alpha} \xi^{3}|q|^{2} \mathrm{~d} x \mathrm{~d} t\right),
$$

where we have used the notation

$$
I_{s, \lambda}(q)=\iint_{Q} \mathrm{e}^{-2 s \alpha}\left((s \xi)^{-1}\left(\left|q_{t}\right|^{2}+|\Delta q|^{2}\right)+s \lambda^{2} \xi|\nabla q|^{2}+s^{3} \lambda^{4} \xi^{3}|q|^{2}\right) \mathrm{d} x \mathrm{~d} t .
$$

This result is a particular case of Lemma 1.2 of Chapter I in [5]. For completeness and also in order to explain and justify the particular form of the constants $\lambda^{*}$ and $s^{*}(\lambda)$, we give a complete proof in the appendix, at the end of this paper. 
Let us continue with the proof of Theorem 1. We can view $\varphi$ as a solution by transposition of (4). This means that $\varphi$ is the unique function in $L^{2}(Q)$ satisfying

$$
\left\{\begin{array}{c}
\iint_{Q} \varphi h \mathrm{~d} x \mathrm{~d} t=\iint_{Q} f_{1}(x, t) z \mathrm{~d} x \mathrm{~d} t-\iint_{Q} f_{2}(x, t) \cdot \nabla z \mathrm{~d} x \mathrm{~d} t \\
+\iint_{\Sigma} f_{3}(x, t) z \mathrm{~d} \sigma \mathrm{d} t+\int_{\Omega} \varphi^{0}(x) z(x, T) \mathrm{d} x \quad \forall h \in L^{2}(Q),
\end{array}\right.
$$

where we have denoted by $z$ the (strong) solution of the following problem:

$$
\begin{cases}z_{t}-\Delta z=h(x, t) & \text { in } Q \\ \frac{\partial z}{\partial n}=0 & \text { on } \Sigma \\ z(x, 0)=0 & \text { in } \Omega .\end{cases}
$$

We will argue as follows. Let us first estimate the second term in the left hand side of (5), i.e.

$$
s^{3} \lambda^{4} \iint_{Q} \mathrm{e}^{-2 s \alpha} \xi^{3}|\varphi|^{2} \mathrm{~d} x \mathrm{~d} t .
$$

To this end, we will deal with techniques inspired by the arguments in [6].

Thus, let us see that the term in (17) can be bounded by the right hand side of (5), i.e.

$$
\begin{aligned}
s^{3} \lambda^{4} \iint_{Q} \mathrm{e}^{-2 s \alpha} \xi^{3}|\varphi|^{2} \mathrm{~d} x \mathrm{~d} t & \leq C(\Omega, \omega)\left(\iint_{Q} \mathrm{e}^{-2 s \alpha}\left|f_{1}\right|^{2} \mathrm{~d} x \mathrm{~d} t\right. \\
+s^{2} \lambda^{2} \iint_{Q} \mathrm{e}^{-2 s \alpha} \xi^{2}\left|f_{2}\right|^{2} \mathrm{~d} x \mathrm{~d} t+s \lambda \iint_{\Sigma} \mathrm{e}^{-2 s \alpha} \xi\left|f_{3}\right|^{2} \mathrm{~d} \sigma \mathrm{d} t & \left.+s^{3} \lambda^{4} \iint_{\omega \times(0, T)} \mathrm{e}^{-2 s \alpha} \xi^{3}|\varphi|^{2} \mathrm{~d} x \mathrm{~d} t\right)
\end{aligned}
$$

for a good choice of the parameters $\lambda$ and $s$.

Let us consider the following constrained extremal problem:

$$
\left\{\begin{array}{l}
\operatorname{Min} \frac{1}{2}\left(\iint_{Q} \mathrm{e}^{2 s \alpha}|z|^{2} \mathrm{~d} x \mathrm{~d} t+s^{-3} \lambda^{-4} \iint_{\omega \times(0, T)} \mathrm{e}^{2 s \alpha} \xi^{-3}|v|^{2} \mathrm{~d} x \mathrm{~d} t\right) \\
\text { subject to } v \in L^{2}(Q) \text { and } \\
\begin{cases}z_{t}-\Delta z=s^{3} \lambda^{4} \mathrm{e}^{-2 s \alpha} \xi^{3} \varphi+v 1_{\omega} & \text { in } Q, \\
\frac{\partial z}{\partial n}=0 & \text { on } \Sigma, \\
z(x, 0)=0, \quad z(x, T)=0 & \text { in } \Omega .\end{cases}
\end{array}\right.
$$

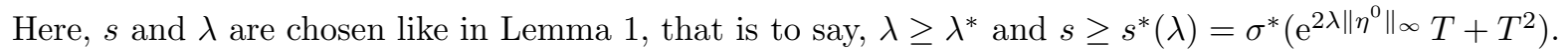

By virtue of Lagrange's principle and arguing as in [6], we are led from (19) to the next optimality system, which is of fourth order in space and second order in time:

$$
\begin{cases}\mathcal{L}\left(\mathrm{e}^{-2 s \alpha} \mathcal{L}^{*} p\right)+s^{3} \lambda^{4} \mathrm{e}^{-2 s \alpha} \xi^{3} p 1_{\omega}=s^{3} \lambda^{4} \mathrm{e}^{-2 s \alpha} \xi^{3} \varphi & \text { in } Q \\ \frac{\partial p}{\partial n}=0, \quad \frac{\partial}{\partial n}\left(\mathrm{e}^{-2 s \alpha} \mathcal{L}^{*} p\right)=0 & \text { on } \Sigma \\ \left(\mathrm{e}^{-2 s \alpha} \mathcal{L}^{*} p\right)_{\mid t=0}=\left(\mathrm{e}^{-2 s \alpha} \mathcal{L}^{*} p\right)_{\mid t=T}=0 & \text { in } \Omega\end{cases}
$$


Here, $\mathcal{L}=\partial_{t}-\Delta$ is the heat operator and $\mathcal{L}^{*}=-\partial_{t}-\Delta$ is its formal adjoint. If $p$ is a solution to (20) (in an appropriate sense), then

$$
\widehat{v}=-s^{3} \lambda^{4} \mathrm{e}^{-2 s \alpha} \xi^{3} p 1_{\omega} \quad \text { and } \quad \widehat{z}=\mathrm{e}^{-2 s \alpha} \mathcal{L}^{*} p
$$

solve (19).

Let us show that (20) has a unique weak solution. To this end, we are going to rewrite this problem as a Lax-Milgram variational equation. Let us introduce the space

$$
X_{0}=\left\{z \in C^{2}(\bar{Q}): \frac{\partial z}{\partial n}=0 \text { on } \Sigma\right\}
$$

and the norm $\|\cdot\|_{X}$, with

$$
\|q\|_{X}^{2}=\iint_{Q} \mathrm{e}^{-2 s \alpha}\left|\mathcal{L}^{*} q\right|^{2} \mathrm{~d} x \mathrm{~d} t+s^{3} \lambda^{4} \iint_{\omega \times(0, T)} \mathrm{e}^{-2 s \alpha} \xi^{3}|q|^{2} \mathrm{~d} x \mathrm{~d} t
$$

for all $q \in X_{0}$.

Due to Lemma $1,\|\cdot\|_{X}$ is indeed a norm in $X_{0}$. Let $X$ be the completion of $X_{0}$ for the norm $\|\cdot\|_{X}$. Then $X$ is a Hilbert space for the scalar product $(\cdot, \cdot)_{X}$, with

$$
(p, q)_{X}=\iint_{Q} \mathrm{e}^{-2 s \alpha}\left(\mathcal{L}^{*} p\right)\left(\mathcal{L}^{*} q\right) \mathrm{d} x \mathrm{~d} t+s^{3} \lambda^{4} \iint_{\omega \times(0, T)} \mathrm{e}^{-2 s \alpha} \xi^{3} p q \mathrm{~d} x \mathrm{~d} t .
$$

With this notation, system (20) is equivalent to find a function $p \in X$ such that

$$
(p, q)_{X}=\ell(q) \quad \forall q \in X
$$

where

$$
\ell(q)=s^{3} \lambda^{4} \iint_{Q} \mathrm{e}^{-2 s \alpha} \xi^{3} \varphi q \mathrm{~d} x \mathrm{~d} t \quad \forall q \in X .
$$

Of course, (22) is equivalent to another extremal problem

$$
\left\{\begin{array}{l}
\text { Minimize } \frac{1}{2}(q, q)_{X}-\ell(q) \\
\text { subject to } q \in X .
\end{array}\right.
$$

By virtue of Lemma 1 , one can easily check that $\ell \in X^{\prime}$. Consequently, one can apply Lax-Milgram lemma and deduce that there exists a unique solution to (20).

Let us now take

$$
h=s^{3} \lambda^{4} \mathrm{e}^{-2 s \alpha} \xi^{3} \varphi+\widehat{v} 1_{\omega}
$$

in (16). This gives

$$
s^{3} \lambda^{4} \iint_{Q} \mathrm{e}^{-2 s \alpha} \xi^{3}|\varphi|^{2} \mathrm{~d} x \mathrm{~d} t=\iint_{Q} f_{1} \widehat{z} \mathrm{~d} x \mathrm{~d} t-\iint_{Q} f_{2} \cdot \nabla \widehat{z} \mathrm{~d} x \mathrm{~d} t+\iint_{\Sigma} f_{3} \widehat{z} \mathrm{~d} \sigma \mathrm{d} t-\iint_{\omega \times(0, T)} \varphi \widehat{v} \mathrm{~d} x \mathrm{~d} t
$$

(recall that $\widehat{v}$ and $\widehat{z}$ are given by (21)). The idea of the proof of (18) is to bound $\widehat{z}, \nabla \widehat{z}$ and $\widehat{v}$ in $Q$ and the trace of $\widehat{z}$ on $\Sigma$ in terms of the left hand side of (23). For this purpose, we first multiply the equation in (20) by $p$ and integrate in $Q$, which gives

$$
\|p\|_{X}^{2} \leq\|\ell\|_{X^{\prime}}\|p\|_{X}
$$


Consequently,

$$
\|p\|_{X}^{2}=\iint_{Q} \mathrm{e}^{2 s \alpha}|\widehat{z}|^{2} \mathrm{~d} x \mathrm{~d} t+s^{-3} \lambda^{-4} \iint_{\omega \times(0, T)} \mathrm{e}^{2 s \alpha} \xi^{-3}|\widehat{v}|^{2} \mathrm{~d} x \mathrm{~d} t \leq C s^{3} \lambda^{4} \iint_{Q} \mathrm{e}^{-2 s \alpha} \xi^{3}|\varphi|^{2} \mathrm{~d} x \mathrm{~d} t
$$

for $\lambda \geq \bar{\lambda}(\Omega, \omega), s \geq \bar{\sigma}(\Omega, \omega)\left(\mathrm{e}^{2 \lambda\left\|\eta^{0}\right\|_{\infty}} T+T^{2}\right)$, since

$$
\|\ell\|_{X^{\prime}} \leq s^{3 / 2} \lambda^{2}\left(\iint_{Q} \mathrm{e}^{-2 s \alpha} \xi^{3}|\varphi|^{2} \mathrm{~d} x \mathrm{~d} t\right)^{1 / 2}
$$

for this choice of the parameters $s$ and $\lambda$. This provides the desired bounds of $\widehat{z}$ and $\widehat{v} 1_{\omega}$.

Let us now multiply the equation satisfied by $\widehat{z}$ by $s^{-2} \lambda^{-2} \mathrm{e}^{2 s \alpha} \xi^{-2} \widehat{z}$ and let us integrate in $Q$. After integration by parts, we obtain:

$$
\begin{aligned}
& \frac{1}{2} s^{-2} \lambda^{-2} \iint_{Q} \mathrm{e}^{2 s \alpha} \xi^{-2} \frac{\partial}{\partial t}|\widehat{z}|^{2} \mathrm{~d} x \mathrm{~d} t+s^{-2} \lambda^{-2} \iint_{Q} \mathrm{e}^{2 s \alpha} \xi^{-2}|\nabla \widehat{z}|^{2} \mathrm{~d} x \mathrm{~d} t-s^{-1} \lambda^{-1} \iint_{Q} \mathrm{e}^{2 s \alpha} \xi^{-1} \nabla \eta^{0} \cdot \nabla|\widehat{z}|^{2} \mathrm{~d} x \mathrm{~d} t \\
& -2 s^{-2} \lambda^{-1} \iint_{Q} \mathrm{e}^{2 s \alpha} \xi^{-2}\left(\nabla \eta^{0} \cdot \nabla \widehat{z}\right) \widehat{z} \mathrm{~d} x \mathrm{~d} t=s \lambda^{2} \iint_{Q} \xi \varphi \widehat{z} \mathrm{~d} x \mathrm{~d} t+s^{-2} \lambda^{-2} \iint_{\omega \times(0, T)} \mathrm{e}^{2 s \alpha} \xi^{-2} \widehat{v} \widehat{z} \mathrm{~d} x \mathrm{~d} t
\end{aligned}
$$

whence

$$
\begin{aligned}
s^{-2} \lambda^{-2} \iint_{Q} & \mathrm{e}^{2 s \alpha} \xi^{-2}|\nabla \widehat{z}|^{2} \mathrm{~d} x \mathrm{~d} t-s^{-1} \lambda^{-1} \iint_{\Sigma} \mathrm{e}^{2 s \alpha} \xi^{-1} \frac{\partial \eta^{0}}{\partial n}|\widehat{z}|^{2} \mathrm{~d} \sigma \mathrm{d} t=\frac{1}{2} s^{-2} \lambda^{-2} \iint_{Q} \frac{\partial}{\partial t}\left(\mathrm{e}^{2 s \alpha} \xi^{-2}\right)|\widehat{z}|^{2} \mathrm{~d} x \mathrm{~d} t \\
& -s^{-1} \lambda^{-1} \iint_{Q} \nabla \cdot\left(\mathrm{e}^{2 s \alpha} \xi^{-1} \nabla \eta^{0}\right)|\widehat{z}|^{2} \mathrm{~d} x \mathrm{~d} t \\
& +2 s^{-2} \lambda^{-1} \iint_{Q} \mathrm{e}^{2 s \alpha} \xi^{-2} \nabla \eta^{0} \cdot \nabla \widehat{z} \widehat{z} \mathrm{~d} x \mathrm{~d} t+s \lambda^{2} \iint_{Q} \xi \varphi \widehat{z} \mathrm{~d} x \mathrm{~d} t \\
& +s^{-2} \lambda^{-2} \iint_{\omega \times(0, T)} \mathrm{e}^{2 s \alpha} \xi^{-2} \widehat{v} \widehat{z} \mathrm{~d} x \mathrm{~d} t .
\end{aligned}
$$

We need now some estimates concerning the weight functions in order to preserve explicit bounds in $s, \lambda$ and $T$. Notice that

$$
\begin{aligned}
\frac{\partial}{\partial t}\left(\mathrm{e}^{2 s \alpha} \xi^{-2}\right) & =-2(T-2 t) \mathrm{e}^{-\lambda \eta^{0}} \mathrm{e}^{2 s \alpha}\left(s \mathrm{e}^{-\lambda \eta^{0}}\left(\mathrm{e}^{2 \lambda\left\|\eta^{0}\right\|_{\infty}}-\mathrm{e}^{\lambda \eta^{0}}\right)-\xi^{-1}\right) \\
& \leq C T \mathrm{e}^{2 s \alpha}\left(\mathrm{e}^{2 \lambda\left\|\eta^{0}\right\|_{\infty}} s+\xi^{-1}\right) \leq C T s \mathrm{e}^{2 s \alpha} \mathrm{e}^{2 \lambda\left\|\eta^{0}\right\|_{\infty},}
\end{aligned}
$$

where we have taken $s \geq C T^{2}$. More generally, observe that, for any fixed $m$, one also has

$$
\left|\nabla\left(\mathrm{e}^{2 s \alpha} \xi^{m}\right)\right| \leq C_{m}(\Omega, \omega) s \lambda \mathrm{e}^{2 s \alpha} \xi^{m+1}
$$

whenever $s \geq C T^{2}$. Indeed, we have

$$
\nabla\left(\mathrm{e}^{2 s \alpha} \xi^{m}\right)=\mathrm{e}^{2 s \alpha} \lambda \nabla \eta^{0} \xi^{m}(2 s \xi+m) \leq C(\Omega, \omega) \mathrm{e}^{2 s \alpha} \lambda \xi^{m}(s \xi+1)
$$

and, taking into account that

we directly get (26).

$$
C s \xi \geq 1 \text { for } s \geq \frac{T^{2}}{4 C}
$$


Turning back to (25), we obtain

$$
\begin{aligned}
& s^{-2} \lambda^{-2} \iint_{Q} \mathrm{e}^{2 s \alpha} \xi^{-2}|\nabla \widehat{z}|^{2} \mathrm{~d} x \mathrm{~d} t-s^{-1} \lambda^{-1} \iint_{\Sigma} \mathrm{e}^{2 s \alpha} \xi^{-1} \frac{\partial \eta^{0}}{\partial n}|\widehat{z}|^{2} \mathrm{~d} \sigma \mathrm{d} t \\
& \leq C(\Omega, \omega)\left(T s^{-1} \lambda^{-2} \mathrm{e}^{2 \lambda\left\|\eta^{0}\right\| \infty} \iint_{Q} \mathrm{e}^{2 s \alpha}|\widehat{z}|^{2} \mathrm{~d} x \mathrm{~d} t+\iint_{Q} \mathrm{e}^{2 s \alpha}|\widehat{z}|^{2} \mathrm{~d} x \mathrm{~d} t+s^{-1} \lambda^{-1} \iint_{Q} \mathrm{e}^{2 s \alpha} \xi^{-1}|\widehat{z}|^{2} \mathrm{~d} x \mathrm{~d} t\right. \\
& +s^{-2} \iint_{Q} \mathrm{e}^{2 s \alpha} \xi^{-2}|\widehat{z}|^{2} \mathrm{~d} x \mathrm{~d} t+s^{2} \lambda^{4} \iint_{Q} \mathrm{e}^{-2 s \alpha} \xi^{2}|\varphi|^{2} \mathrm{~d} x \mathrm{~d} t \\
& \left.\quad+s^{-4} \lambda^{-4} \iint_{Q} \mathrm{e}^{2 s \alpha} \xi^{-4}|\widehat{v}|^{2} \mathrm{~d} x \mathrm{~d} t\right)+\frac{1}{2} s^{-2} \lambda^{-2} \iint_{Q} \mathrm{e}^{2 s \alpha} \xi^{-2}|\nabla \widehat{z}|^{2} \mathrm{~d} x \mathrm{~d} t
\end{aligned}
$$

where we have taken $s \geq C T^{2}$. Now, we take into account (27) and we deduce that

$$
\begin{aligned}
s^{-2} \lambda^{-2} \iint_{Q} \mathrm{e}^{2 s \alpha} \xi^{-2}|\nabla \widehat{z}|^{2} \mathrm{~d} x \mathrm{~d} t-s^{-1} \lambda^{-1} \iint_{\Sigma} \mathrm{e}^{2 s \alpha} \xi^{-1} \frac{\partial \eta^{0}}{\partial n}|\widehat{z}|^{2} \mathrm{~d} \sigma \mathrm{d} t \\
\quad \leq C(\Omega, \omega)\left(\iint_{Q} \mathrm{e}^{2 s \alpha}|\widehat{z}|^{2} \mathrm{~d} x \mathrm{~d} t+s^{3} \lambda^{4} \iint_{Q} \mathrm{e}^{-2 s \alpha} \xi^{3}|\varphi|^{2} \mathrm{~d} x \mathrm{~d} t s^{-3} \lambda^{-4} \iint_{Q} \mathrm{e}^{2 s \alpha} \xi^{-3}|\widehat{v}|^{2} \mathrm{~d} x \mathrm{~d} t\right)
\end{aligned}
$$

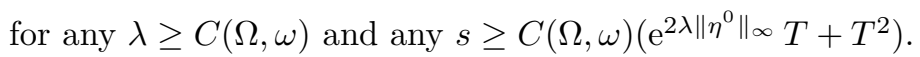

From (14), this gives an estimate of the gradient and the trace of $\widehat{z}$ in terms of $\widehat{z}, \widehat{v} 1_{\omega}$ and $\varphi$. In view of (24), we now have

$$
\begin{aligned}
\iint_{Q} \mathrm{e}^{2 s \alpha}|\widehat{z}|^{2} \mathrm{~d} x \mathrm{~d} t+s^{-2} \lambda^{-2} \iint_{Q} \mathrm{e}^{2 s \alpha} \xi^{-2}|\nabla \widehat{z}|^{2} \mathrm{~d} x \mathrm{~d} t+s^{-1} \lambda^{-1} \iint_{\Sigma} \mathrm{e}^{2 s \alpha} \xi^{-1}|\widehat{z}|^{2} \mathrm{~d} \sigma \mathrm{d} t \\
\quad+s^{-3} \lambda^{-4} \iint_{\omega \times(0, T)} \mathrm{e}^{2 s \alpha} \xi^{-3}|\widehat{v}|^{2} \mathrm{~d} x \mathrm{~d} t \leq C(\Omega, \omega) s^{3} \lambda^{4} \iint_{Q} \mathrm{e}^{-2 s \alpha} \xi^{3}|\varphi|^{2} \mathrm{~d} x \mathrm{~d} t
\end{aligned}
$$

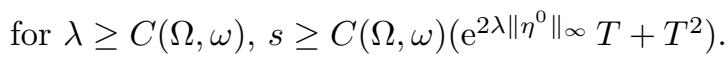

It suffices to combine this inequality and the identity (23) to deduce (18).

Let us now show that

$$
\begin{aligned}
& s \lambda^{2} \iint_{Q} \mathrm{e}^{-2 s \alpha} \xi|\nabla \varphi|^{2} \mathrm{~d} x \mathrm{~d} t \leq C(\Omega, \omega)\left(\iint_{Q} \mathrm{e}^{-2 s \alpha}\left|f_{1}\right|^{2} \mathrm{~d} x \mathrm{~d} t\right. \\
& \left.\quad+s^{2} \lambda^{2} \iint_{Q} \mathrm{e}^{-2 s \alpha} \xi^{2}\left|f_{2}\right|^{2} \mathrm{~d} x \mathrm{~d} t+s \lambda \iint_{\Sigma} \mathrm{e}^{-2 s \alpha} \xi\left|f_{3}\right|^{2} \mathrm{~d} \sigma \mathrm{d} t+s^{3} \lambda^{4} \iint_{\omega \times(0, T)} \mathrm{e}^{-2 s \alpha} \xi^{3}|\varphi|^{2} \mathrm{~d} x \mathrm{~d} t\right)
\end{aligned}
$$

To this end, we now have to use not only that $\varphi$ is a solution by transposition but a weak solution as well. More precisely, let us take

$$
v=s \lambda^{2} \mathrm{e}^{-2 s \alpha(\cdot, t)} \xi(\cdot, t) \varphi(\cdot, t)
$$

in (12). Then, let us integrate in $(0, T)$ and let us perform integrations by parts similarly as we did before. We get:

$$
\begin{aligned}
-\frac{1}{2} s \lambda^{2} \iint_{Q} \mathrm{e}^{-2 s \alpha} \xi & \frac{\partial}{\partial t}|\varphi|^{2} \mathrm{~d} x \mathrm{~d} t+s \lambda^{2} \iint_{Q} \mathrm{e}^{-2 s \alpha} \xi|\nabla \varphi|^{2} \mathrm{~d} x \mathrm{~d} t+s \lambda^{2} \iint_{Q} \nabla \varphi \cdot \nabla\left(\mathrm{e}^{-2 s \alpha} \xi\right) \varphi \mathrm{d} x \mathrm{~d} t \\
= & s \lambda^{2} \iint_{Q} \mathrm{e}^{-2 s \alpha} \xi f_{1} \varphi \mathrm{d} x \mathrm{~d} t-s \lambda^{2} \iint_{Q} f_{2} \cdot \nabla\left(\mathrm{e}^{-2 s \alpha} \xi \varphi\right) \mathrm{d} x \mathrm{~d} t+s \lambda^{2} \iint_{\Sigma} \mathrm{e}^{-2 s \alpha} \xi f_{3} \varphi \mathrm{d} \sigma \mathrm{d} t
\end{aligned}
$$


We integrate by parts again and we obtain

$$
\begin{aligned}
& s \lambda^{2} \iint_{Q} \mathrm{e}^{-2 s \alpha} \xi|\nabla \varphi|^{2} \mathrm{~d} x \mathrm{~d} t=-\frac{1}{2} s \lambda^{2} \iint_{Q}\left(\mathrm{e}^{-2 s \alpha} \xi\right)_{t}|\varphi|^{2} \mathrm{~d} x \mathrm{~d} t-s \lambda^{2} \iint_{Q} \nabla \varphi \cdot \nabla\left(\mathrm{e}^{-2 s \alpha} \xi\right) \varphi \mathrm{d} x \mathrm{~d} t \\
& +s \lambda^{2} \iint_{Q} \mathrm{e}^{-2 s \alpha} \xi f_{1} \varphi \mathrm{d} x \mathrm{~d} t-s \lambda^{2} \iint_{Q} f_{2} \cdot \nabla\left(\mathrm{e}^{-2 s \alpha} \xi\right) \varphi \mathrm{d} x \mathrm{~d} t \\
& -s \lambda^{2} \iint_{Q} f_{2} \cdot \nabla \varphi \mathrm{e}^{-2 s \alpha} \xi \mathrm{d} x \mathrm{~d} t+s \lambda^{2} \iint_{\Sigma} \mathrm{e}^{-2 s \alpha} \xi f_{3} \varphi \mathrm{d} \sigma \mathrm{d} t
\end{aligned}
$$

In view of $(26)$, we find:

$$
\begin{aligned}
& s \lambda^{2} \iint_{Q} \mathrm{e}^{-2 s \alpha} \xi|\nabla \varphi|^{2} \mathrm{~d} x \mathrm{~d} t \leq C(\Omega, \omega)\left(T s^{2} \lambda^{2} \mathrm{e}^{2 \lambda\left\|\eta^{0}\right\|_{\infty}} \iint_{Q} \mathrm{e}^{-2 s \alpha} \xi^{3}|\varphi|^{2} \mathrm{~d} x d t+s^{3} \lambda^{4} \iint_{Q} \mathrm{e}^{-2 s \alpha} \xi^{3}|\varphi|^{2} \mathrm{~d} x \mathrm{~d} t\right. \\
& +\iint_{Q} \mathrm{e}^{-2 s \alpha}\left|f_{1}\right|^{2} \mathrm{~d} x \mathrm{~d} t+s^{2} \lambda^{4} \iint_{Q} \mathrm{e}^{-2 s \alpha} \xi^{2}|\varphi|^{2} \mathrm{~d} x \mathrm{~d} t+s \lambda^{2} \iint_{Q} \mathrm{e}^{-2 s \alpha} \xi\left|f_{2}\right|^{2} \mathrm{~d} x \mathrm{~d} t \\
& \left.s \lambda \iint_{\Sigma} \mathrm{e}^{-2 s \alpha} \xi\left|f_{3}\right|^{2} \mathrm{~d} \sigma \mathrm{d} t+s \lambda^{3} \iint_{\Sigma} \mathrm{e}^{-2 s \alpha} \xi|\varphi|^{2} \mathrm{~d} \sigma \mathrm{d} t\right)+\frac{1}{2} s \lambda^{2} \iint_{Q} \mathrm{e}^{-2 s \alpha} \xi|\nabla \varphi|^{2} \mathrm{~d} x \mathrm{~d} t
\end{aligned}
$$

where we have taken $s \geq C T^{2}$ and $\lambda \geq C$. Making several simplifications, we easily see that

$$
\left\{\begin{array}{c}
s \lambda^{2} \iint_{Q} \mathrm{e}^{-2 s \alpha} \xi|\nabla \varphi|^{2} \mathrm{~d} x \mathrm{~d} t \leq C\left(s^{3} \lambda^{4} \iint_{Q} \mathrm{e}^{-2 s \alpha} \xi^{3}|\varphi|^{2} \mathrm{~d} x \mathrm{~d} t\right. \\
\quad+\iint_{Q} \mathrm{e}^{-2 s \alpha}\left|f_{1}\right|^{2} \mathrm{~d} x \mathrm{~d} t+s^{2} \lambda^{2} \iint_{Q} \mathrm{e}^{-2 s \alpha} \xi^{2}\left|f_{2}\right|^{2} \mathrm{~d} x \mathrm{~d} t \\
\left.\quad+s \lambda \iint_{\Sigma} \mathrm{e}^{-2 s \alpha} \xi\left|f_{3}\right|^{2} \mathrm{~d} \sigma \mathrm{d} t+s \lambda^{3} \iint_{\Sigma} \mathrm{e}^{-2 s \alpha} \xi|\varphi|^{2} \mathrm{~d} \sigma d t\right)
\end{array}\right.
$$

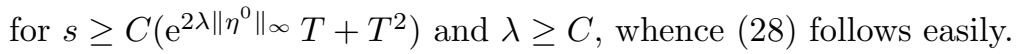

Let us finally estimate the trace of $\varphi$ in terms of $\varphi$ and $\nabla \varphi$. Notice that

$$
\begin{aligned}
-s^{2} \lambda^{3} \iint_{Q} \mathrm{e}^{-2 s \alpha} \xi^{2}\left(\nabla \eta^{0} \cdot \nabla \varphi\right) \varphi \mathrm{d} x \mathrm{~d} t=-\frac{1}{2} s^{2} \lambda^{3} \iint_{\Sigma} \mathrm{e}^{-2 s \alpha} \xi^{2} \frac{\partial \eta^{0}}{\partial n}|\varphi|^{2} \mathrm{~d} \sigma \mathrm{d} t & +\frac{1}{2} s^{2} \lambda^{3} \iint_{Q} \nabla \cdot\left(\mathrm{e}^{-2 s \alpha} \xi^{2} \nabla \eta^{0}\right)|\varphi|^{2} \mathrm{~d} x \mathrm{~d} t
\end{aligned}
$$

Taking into account (14), the following is found:

$$
\begin{aligned}
& s^{2} \lambda^{3} \iint_{\Sigma} \mathrm{e}^{-2 s \alpha} \xi^{2}|\varphi|^{2} \mathrm{~d} \sigma \mathrm{d} t \leq C s^{2} \lambda^{3} \iint_{Q}\left|\nabla \cdot\left(\mathrm{e}^{-2 s \alpha} \xi^{2} \nabla \eta^{0}\right)\right||\varphi|^{2} \mathrm{~d} x \mathrm{~d} t \\
& +C\left(s^{3} \lambda^{4} \iint_{Q} \mathrm{e}^{-2 s \alpha} \xi^{3}|\varphi|^{2} \mathrm{~d} x \mathrm{~d} t+s \lambda^{2} \iint_{Q} \mathrm{e}^{-2 s \alpha} \xi|\nabla \varphi|^{2} \mathrm{~d} x \mathrm{~d} t\right) \\
& \leq C\left(s^{3} \lambda^{4} \iint_{Q} \mathrm{e}^{-2 s \alpha} \xi^{3}|\varphi|^{2} \mathrm{~d} x \mathrm{~d} t+s \lambda^{2} \iint_{Q} \mathrm{e}^{-2 s \alpha} \xi|\nabla \varphi|^{2} \mathrm{~d} x \mathrm{~d} t\right)
\end{aligned}
$$

with $s \geq C\left(T+T^{2}\right)$ and $\lambda \geq C$.

This last inequality, together with (18) and (29), provides (5) and permits to achieve the proof of Theorem 1. 


\section{Controllability of the linear system}

This section is devoted to prove Theorem 2. This will be a consequence of the Carleman inequality (5). We will start with an explicit bound of the weak solution to the linear problem

$$
\begin{cases}y_{t}-\Delta y+B(x, t) \cdot \nabla y+a(x, t) y=f(x, t) & \text { in } Q, \\ \frac{\partial y}{\partial n}+\beta(x, t) y=0 & \text { on } \Sigma, \\ y(x, 0)=y^{0}(x) & \text { in } \Omega,\end{cases}
$$

where $f \in L^{2}(Q), y^{0} \in L^{2}(\Omega)$ and (2) is fulfilled. Then, we will use this result in combination with (5) to deduce the observability inequality (8) for the solutions to (6). Finally, we will end the proof of theorem 2 in a classical way, using this observability inequality.

Proposition 1. Under the previous assumptions, the weak solution to (30) satisfies the estimate

$$
\|y\|_{Y} \leq \mathrm{e}^{C T\left(1+\|a\|_{\infty}+\|B\|_{\infty}^{2}+\|\beta\|_{\infty}^{2}\right)}\left(\|f\|_{L^{2}(Q)}+\left\|y^{0}\right\|_{L^{2}(\Omega)}\right)
$$

for some constant $C>0$. Here, $Y$ is the usual energy space:

$$
Y=L^{2}\left(0, T ; H^{1}(\Omega)\right) \cap C^{0}\left([0, T] ; L^{2}(\Omega)\right)
$$

Proof. The existence and uniqueness of a solution to (30) is well known. Furthermore, the following identity can be deduced for each $t \in(0, T)$ in a standard way:

$$
\begin{aligned}
\frac{1}{2} \frac{\mathrm{d}}{\mathrm{d} t} \int_{\Omega}|y(x, t)|^{2} \mathrm{~d} x+\int_{\Omega} \mid & \left.\nabla y(x, t)\right|^{2} \mathrm{~d} x+\int_{\partial \Omega} \beta(x, t)|y(x, t)|^{2} \mathrm{~d} \sigma \\
& +\int_{\Omega} B(x, t) \cdot \nabla y(x, t) y(x, t) \mathrm{d} x+\int_{\Omega} a(x, t)|y(x, t)|^{2} \mathrm{~d} x=\int_{\Omega} f(x, t) y(x, t) \mathrm{d} x
\end{aligned}
$$

We will now use the following trace estimate for the functions in $H^{1}(\Omega)$ :

$$
\left\{\begin{array}{l}
\int_{\partial \Omega}|u|^{2} \mathrm{~d} \sigma \leq C\left(\int_{\Omega}\left(|u|^{2}+|\nabla u|^{2}\right) \mathrm{d} x\right)^{1 / 2}\left(\int_{\Omega}|u|^{2} \mathrm{~d} x\right)^{1 / 2} \\
\forall u \in H^{1}(\Omega)
\end{array}\right.
$$

for some positive $C=C(\Omega)$. This inequality can be proved arguing first for regular functions in a dense subspace of $H^{1}(\Omega)$ and then passing to the limit. For a regular function $u,(33)$ is very easy to establish when $\Omega=\mathbb{R}_{+}^{N}$. Then, a standard localization argument leads to the proof in the case of a general domain $\Omega$.

In view of (32) and (33), we have:

$$
\begin{aligned}
\frac{1}{2} \frac{\mathrm{d}}{\mathrm{d} t} \int_{\Omega}|y(x, t)|^{2} \mathrm{~d} x+\int_{\Omega}|\nabla y(x, t)|^{2} \mathrm{~d} x \leq-\int_{\Omega} B(x, t) \cdot \nabla y(x, t) y(x, t) \mathrm{d} x \\
\quad-\int_{\Omega} a(x, t)|y(x, t)|^{2} \mathrm{~d} x+\int_{\Omega} f(x, t) y(x, t) \mathrm{d} x+C\|\beta\|_{\infty}\|y(\cdot, t)\|_{H^{1}(\Omega)}\|y(\cdot, t)\|_{L^{2}(\Omega)} .
\end{aligned}
$$


Combining this and Young's inequality, we obtain:

$$
\frac{\mathrm{d}}{\mathrm{d} t}\|y(\cdot, t)\|_{L^{2}(\Omega)}^{2}+\|y(\cdot, t)\|_{H^{1}(\Omega)}^{2} \leq C\left(\left(1+\|a\|_{\infty}+\|B\|_{\infty}^{2}+\|\beta\|_{\infty}^{2}\right)\|y(\cdot, t)\|_{L^{2}(\Omega)}^{2}+\|f(\cdot, t)\|_{L^{2}(\Omega)}^{2}\right)
$$

for all $t \in(0, T)$. From these estimates, it is not difficult to obtain (31).

This ends the proof.

The announced observability estimate is proved in the following result:

Proposition 2. For every $\varphi^{0} \in L^{2}(\Omega)$, the associated solution to (6) satisfies the observability inequality

$$
\iint_{\Omega \times(T / 4,3 T / 4)}|\varphi|^{2} \mathrm{~d} x \mathrm{~d} t \leq K \iint_{\omega \times(0, T)}|\varphi|^{2} \mathrm{~d} x \mathrm{~d} t
$$

for a constant $K$ of the form

$$
K=\exp \left\{C\left(1+\frac{1}{T}+\|a\|_{\infty}^{2 / 3}+\|B\|_{\infty}^{2}+\|\beta\|_{\infty}^{2}\right)\right\}
$$

Proof. Let $\varphi^{0} \in L^{2}(\Omega)$ be given. Notice that the corresponding $\varphi$ solves (4) with

$$
f_{1}=-a \varphi \in L^{2}(Q), \quad f_{2}=\varphi B \in L^{2}\left(0, T ; L^{2}(\Omega)^{N}\right), \quad f_{3}=-\beta \varphi \in L^{2}(\Sigma)
$$

Thus, we can apply theorem 1 to $\varphi$ and deduce that

$$
\begin{aligned}
\iint_{Q} \mathrm{e}^{-2 s \alpha}\left(s \lambda^{2} \xi|\nabla \varphi|^{2}+s^{3} \lambda^{4} \xi^{3}|\varphi|^{2}\right) \mathrm{d} x \mathrm{~d} t+s^{2} \lambda^{3} \iint_{\Sigma} \mathrm{e}^{-2 s \alpha} \xi^{2}|\varphi|^{2} \mathrm{~d} \sigma \mathrm{d} t \\
\leq C(\Omega, \omega)\left(\|a\|_{\infty}^{2} \iint_{Q} \mathrm{e}^{-2 s \alpha}|\varphi|^{2} \mathrm{~d} x \mathrm{~d} t+s^{2} \lambda^{2}\|B\|_{\infty}^{2} \iint_{Q} \mathrm{e}^{-2 s \alpha} \xi^{2}|\varphi|^{2} \mathrm{~d} x \mathrm{~d} t\right. \\
\left.\quad+s \lambda\|\beta\|_{\infty}^{2} \iint_{\Sigma} \mathrm{e}^{-2 s \alpha} \xi|\varphi|^{2} \mathrm{~d} \sigma \mathrm{d} t+s^{3} \lambda^{4} \iint_{\omega \times(0, T)} \mathrm{e}^{-2 s \alpha} \xi^{3}|\varphi|^{2} \mathrm{~d} x \mathrm{~d} t\right)
\end{aligned}
$$

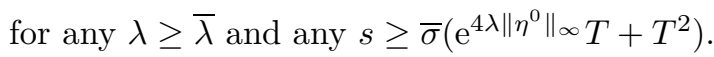

We will now try to eliminate the global terms in the right hand side of this inequality by making a convenient choice of the parameter $s$.

Taking $s \geq C T^{2}\left(\|a\|_{\infty}^{2 / 3}+\|B\|_{\infty}^{2}\right)$, we see that

$$
C\left(s^{2} \lambda^{2}\|B\|_{\infty}^{2} \iint_{Q} \mathrm{e}^{-2 s \alpha} \xi^{2}|\varphi|^{2} \mathrm{~d} x \mathrm{~d} t+\|a\|_{\infty}^{2} \iint_{Q} \mathrm{e}^{-2 s \alpha}|\varphi|^{2} \mathrm{~d} x \mathrm{~d} t\right) \leq \frac{1}{2} s^{3} \lambda^{4} \iint_{Q} \mathrm{e}^{-2 s \alpha} \xi^{3}|\varphi|^{2} \mathrm{~d} x \mathrm{~d} t
$$

On the other hand, taking $s \geq C T^{2}\|\beta\|_{\infty}^{2}$, we find that

$$
C s \lambda\|\beta\|_{\infty}^{2} \iint_{\Sigma} \mathrm{e}^{-2 s \alpha} \xi|\varphi|^{2} \mathrm{~d} \sigma \mathrm{d} t \leq \frac{1}{2} s^{2} \lambda^{3} \iint_{\Sigma} \mathrm{e}^{-2 s \alpha} \xi^{2}|\varphi|^{2} \mathrm{~d} \sigma \mathrm{d} t
$$


All this leads to the estimate

$$
\iint_{Q} \mathrm{e}^{-2 s \alpha} \xi^{3}|\varphi|^{2} \mathrm{~d} x \mathrm{~d} t \leq C \iint_{\omega \times(0, T)} \mathrm{e}^{-2 s \alpha} \xi^{3}|\varphi|^{2} \mathrm{~d} x \mathrm{~d} t,
$$

which holds for $\lambda \geq \bar{\lambda}$ and $s \geq \bar{\sigma}\left(\mathrm{e}^{4 \lambda\left\|\eta^{0}\right\|_{\infty}} T+T^{2}\left(1+\|a\|_{\infty}^{2 / 3}+\|B\|_{\infty}^{2}+\|\beta\|_{\infty}^{2}\right)\right)$.

Taking into account the properties of the weight functions as well as the choice of $s$ and $\lambda$ we have made, it is not difficult to realize that the function

$$
t \mapsto \exp \left(-2 s \max _{x \in \bar{\Omega}} \alpha(t)\right) \min _{x \in \bar{\Omega}} \xi(t)^{3}
$$

reaches its minimum in $(T / 4,3 T / 4)$ at $t=T / 4$ and that the function

$$
t \mapsto \exp \left(-2 s \min _{x \in \bar{\Omega}} \alpha(t)\right) \max _{x \in \bar{\Omega}} \xi(t)^{3}
$$

reaches its maximum in $(0, T)$ at $t=T / 2$. With this, the previous Carleman inequality directly gives

$$
\begin{aligned}
\iint_{\Omega \times(T / 4,3 T / 4)}|\varphi|^{2} \mathrm{~d} x \mathrm{~d} t \leq \exp \left\{-2 s\left(\min _{x \in \bar{\Omega}} \alpha\left(x, \frac{T}{2}\right)\right.\right. & \left.\left.-\max _{x \in \bar{\Omega}} \alpha\left(x, \frac{T}{4}\right)\right)\right\} \\
& \times \min _{x \in \bar{\Omega}} \xi\left(x, \frac{T}{4}\right)^{-3} \max _{x \in \bar{\Omega}} \xi\left(x, \frac{T}{2}\right)^{3} \iint_{\omega \times(0, T)}|\varphi|^{2} \mathrm{~d} x \mathrm{~d} t
\end{aligned}
$$

for the same choice of the parameters $s$ and $\lambda$.

Now, taking $\lambda=\bar{\lambda}$ and $s=\bar{s}=\bar{\sigma}\left(\mathrm{e}^{4 \bar{\lambda}\left\|\eta^{0}\right\|_{\infty}} T+T^{2}\left(1+\|a\|_{\infty}^{2 / 3}+\|B\|_{\infty}^{2}+\|\beta\|_{\infty}^{2}\right)\right)$, we have

$$
\iint_{\Omega \times(T / 4,3 T / 4)}|\varphi|^{2} \mathrm{~d} x \mathrm{~d} t \leq C(\Omega, \omega) \mathrm{e}^{C(\Omega, \omega) \bar{s} / T^{2}} \iint_{\omega \times(0, T)}|\varphi|^{2} \mathrm{~d} x \mathrm{~d} t
$$

which gives (35) and (34).

This ends the proof of Proposition 2.

Let us now finish the proof of Theorem 2. We will apply a well known argument that has already been used in several similar situations (see $[3,5])$.

Let us introduce a function $\eta \in C^{\infty}(0, T)$, with

$$
\eta(t)=1 \text { for } t \in(0, T / 4), \quad \eta(t)=0 \text { for } t \in(3 T / 4, T)
$$

and

$$
\left|\eta^{\prime}(t)\right| \leq C / T \text { for } t \in(0, T) .
$$

Let $\chi$ be the weak solution of

$$
\begin{cases}\chi_{t}-\Delta \chi+B(x, t) \cdot \nabla \chi+a(x, t) \chi=0 & \text { in } Q \\ \frac{\partial \chi}{\partial n}+\beta(x, t) \chi=0 & \text { on } \Sigma \\ \chi(x, 0)=y^{0}(x) & \text { in } \Omega .\end{cases}
$$


and let us put $y=w+\eta \chi$. If $y$ is the state associated to $v$, i.e. the solution to (1), then $w$ satisfies

$$
\begin{cases}w_{t}-\Delta w+B(x, t) \cdot \nabla w+a(x, t) w=-\eta^{\prime}(t) \chi+v 1_{\omega} & \text { in } Q, \\ \frac{\partial w}{\partial n}+\beta(x, t) w=0 & \text { on } \Sigma, \\ w(x, 0)=0 & \text { in } \Omega .\end{cases}
$$

Our task is to find a control $v \in L^{2}(\omega \times(0, T))$ such that the associated solution to (36) satisfies

$$
w(x, T)=0 \quad \text { in } \Omega .
$$

After this, just taking $y=w+\eta \chi$ we will have proved our result with a control in $L^{2}(\omega \times(0, T))$.

For each $\varepsilon>0$, let us consider the functional $J_{\varepsilon}$, with

$$
\left\{\begin{array}{l}
J_{\varepsilon}\left(\varphi^{0}\right)=\frac{1}{2} \iint_{\omega \times(0, T)}|\varphi|^{2} \mathrm{~d} x \mathrm{~d} t+\varepsilon\left\|\varphi^{0}\right\|_{L^{2}(\Omega)}-\iint_{Q} \eta^{\prime} \chi \varphi \mathrm{d} x \mathrm{~d} t \\
\forall \varphi^{0} \in L^{2}(\Omega),
\end{array}\right.
$$

where, for each $\varphi^{0} \in L^{2}(\Omega), \varphi$ is the solution to (6) associated to $\varphi^{0}$.

It is clear that

$$
\varphi^{0} \mapsto J_{\varepsilon}\left(\varphi^{0}\right)
$$

is a continuous, strictly convex and (in view of (8)) coercive function on $L^{2}(\Omega)$. Consequently, it possesses exactly one minimizer $\varphi_{\varepsilon}^{0}$ and it is not difficult to check that $\varphi_{\varepsilon}^{0}=0$ if and only if the solution $\tilde{w}$ to $(36)$ associated to $v=0$ satisfies $\|\tilde{w}(\cdot, T)\|_{L^{2}(\Omega)} \leq \varepsilon$.

Let us denote by $\varphi_{\varepsilon}$ the solution to (6) associated to $\varphi_{\varepsilon}^{0}$, let us put

$$
v_{\varepsilon}=\varphi_{\varepsilon} 1_{\omega}
$$

and let us denote by $w_{\varepsilon}$ the solution to (36) associated to the control $v_{\varepsilon}$. Then

$$
\left\|w_{\varepsilon}(\cdot, T)\right\|_{L^{2}(\Omega)} \leq \varepsilon
$$

Indeed, it is not restrictive to assume that $\varphi_{\varepsilon}^{0} \neq 0$. Accordingly, $J_{\varepsilon}$ is differentiable at $\varphi_{\varepsilon}^{0}$ and

$$
\left(J_{\varepsilon}^{\prime}\left(\varphi_{\varepsilon}^{0}\right), \varphi^{0}\right)_{L^{2}(\Omega)}=0 \quad \forall \varphi^{0} \in L^{2}(\Omega) .
$$

That is to say,

$$
\left\{\begin{array}{l}
\iint_{\omega \times(0, T)} \varphi_{\varepsilon} \varphi \mathrm{d} x \mathrm{~d} t+\left(\varepsilon \frac{\varphi_{\varepsilon}^{0}}{\left\|\varphi_{\varepsilon}^{0}\right\|_{L^{2}}}, \varphi^{0}\right)_{L^{2}(\Omega)}-\iint_{Q} \eta^{\prime} \chi \varphi \mathrm{d} x \mathrm{~d} t=0 \\
\forall \varphi^{0} \in L^{2}(\Omega) .
\end{array}\right.
$$

Since

$$
\iint_{\omega \times(0, T)} \varphi_{\varepsilon} \varphi \mathrm{d} x \mathrm{~d} t-\iint_{Q} \eta^{\prime} \chi \varphi \mathrm{d} x \mathrm{~d} t=\left(w_{\varepsilon}(\cdot, T), \varphi^{0}\right)_{L^{2}(\Omega)},
$$

we have

$$
\left(w_{\varepsilon}(\cdot, T), \varphi^{0}\right)_{L^{2}(\Omega)}=-\left(\varepsilon \frac{\varphi_{\varepsilon}^{0}}{\left\|\varphi_{\varepsilon}^{0}\right\|_{L^{2}(\Omega)}}, \varphi^{0}\right)_{L^{2}(\Omega)} \quad \forall \varphi^{0} \in L^{2}(\Omega),
$$

which implies (38). 
Since $J_{\varepsilon}\left(\varphi_{\varepsilon}^{0}\right) \leq J_{\varepsilon}(0)=0$, we also have

$$
\left\|v_{\varepsilon}\right\|_{L^{2}(\omega \times(0, T))}^{2} \leq\left(\iint_{\Omega \times(T / 4,3 T / 4)}\left|\varphi_{\varepsilon}\right|^{2} \mathrm{~d} x \mathrm{~d} t\right)^{1 / 2}\left(\iint_{\Omega \times(T / 4,3 T / 4)}\left|\eta^{\prime} \chi\right|^{2} \mathrm{~d} x \mathrm{~d} t\right)^{1 / 2}
$$

From Proposition 2 and the definition of $v_{\varepsilon}$, we deduce now that

$$
\left\|v_{\varepsilon}\right\|_{L^{2}(\omega \times(0, T))}^{2} \leq \frac{C}{T} K^{1 / 2}\left\|v_{\varepsilon}\right\|_{L^{2}(Q)}\left(\iint_{\Omega \times(T / 4,3 T / 4)}|\chi|^{2} \mathrm{~d} x \mathrm{~d} t\right)^{1 / 2}
$$

and, using Proposition 1, we have

$$
\left\|v_{\varepsilon}\right\|_{L^{2}(\omega \times(0, T))} \leq C K^{1 / 2}\|\chi\|_{Y} \leq H\left\|y^{0}\right\|_{L^{2}(\Omega)}
$$

where the constant $H$ is as in (11).

Consequently, $v_{\varepsilon} 1_{\omega}$ and $w_{\varepsilon}$ are uniformly bounded in the spaces $L^{2}(\omega \times(0, T))$ and

$$
Z=\left\{w \in L^{2}\left(0, T ; H^{1}(\Omega)\right): w_{t} \in L^{2}\left(0, T ; H^{-1}(\Omega)\right)\right\}
$$

respectively. Obviously, we can extract sequences converging weakly to a control $v 1_{\omega}$ and the associated solution $w$ of $(36)$, with

$$
w(x, T)=0 \text { in } \Omega .
$$

We have thus proved the existence of a control $v \in L^{2}(Q)$ such that (10) and (37) are fulfilled.

This ends the proof of Theorem 2.

\section{Appendix: Proof of Lemma 1}

We divide the proof in three steps:

1 - First, we set $\psi=\mathrm{e}^{-s \alpha} q$ and we prove the following inequality:

$$
\begin{aligned}
& \iint_{Q}\left(s^{-1} \xi^{-1}\left(\left|\psi_{t}\right|^{2}+|\Delta \psi|^{2}\right)+s \lambda^{2} \xi|\nabla \psi|^{2}+s^{3} \lambda^{4} \xi^{3}|\psi|^{2}\right) \mathrm{d} x \mathrm{~d} t \\
& -2 s^{3} \lambda^{3} \iint_{\Sigma}\left|\nabla \eta^{0}\right|^{2} \xi^{3} \frac{\partial \eta^{0}}{\partial n}|\psi|^{2} \mathrm{~d} \sigma \mathrm{d} t-4 s \lambda^{2} \iint_{\Sigma}\left|\nabla \eta^{0}\right|^{2} \xi \frac{\partial \psi}{\partial n} \psi \mathrm{d} \sigma \mathrm{d} t \\
& -4 s \lambda \iint_{\Sigma} \frac{\partial \eta^{0}}{\partial n} \xi\left|\frac{\partial \psi}{\partial n}\right|^{2} \mathrm{~d} \sigma \mathrm{d} t+2 s \lambda \iint_{\Sigma} \frac{\partial \eta^{0}}{\partial n} \xi|\nabla \psi|^{2} \mathrm{~d} \sigma \mathrm{d} t \\
& +2 \iint_{\Sigma} \frac{\partial \psi}{\partial n} \psi_{t} \mathrm{~d} \sigma \mathrm{d} t-2 s^{2} \lambda \iint_{\Sigma} \alpha_{t} \frac{\partial \eta^{0}}{\partial n} \xi|\psi|^{2} \mathrm{~d} \sigma \mathrm{d} t \\
& \leq C\left(\iint_{Q} \mathrm{e}^{-2 s \alpha}|f|^{2} \mathrm{~d} x \mathrm{~d} t+s^{3} \lambda^{4} \iint_{\omega \times(0, T)} \xi^{3}|\psi|^{2} \mathrm{~d} x \mathrm{~d} t\right)
\end{aligned}
$$

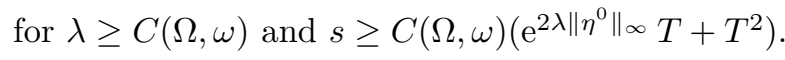


2 - Then, we set $\tilde{\psi}=\mathrm{e}^{-s \tilde{\alpha}} q$ and we prove that

$$
\begin{aligned}
& \iint_{Q}\left(s ^ { - 1 } \tilde { \xi } ^ { - 1 } \left(\left|\tilde{\psi}_{t}\right|^{2}+\right.\right.\left.\left.|\Delta \tilde{\psi}|^{2}\right)+s \lambda^{2} \tilde{\xi}|\nabla \tilde{\psi}|^{2}+s^{3} \lambda^{4} \tilde{\xi}^{3}|\tilde{\psi}|^{2}\right) \mathrm{d} x \mathrm{~d} t \\
&+2 s^{3} \lambda^{3} \iint_{\Sigma}\left|\nabla \eta^{0}\right|^{2} \tilde{\xi}^{3} \frac{\partial \eta^{0}}{\partial n}|\tilde{\psi}|^{2} \mathrm{~d} \sigma \mathrm{d} t-4 s \lambda^{2} \iint_{\Sigma}\left|\nabla \eta^{0}\right|^{2} \tilde{\xi} \frac{\partial \tilde{\psi}}{\partial n} \tilde{\psi} \mathrm{d} \sigma \mathrm{d} t \\
&+4 s \lambda \iint_{\Sigma} \frac{\partial \eta^{0}}{\partial n} \tilde{\xi}\left|\frac{\partial \tilde{\psi}}{\partial n}\right|^{2} \mathrm{~d} \sigma \mathrm{d} t-2 s \lambda \iint_{\Sigma} \frac{\partial \eta^{0}}{\partial n} \tilde{\xi}|\nabla \tilde{\psi}|^{2} \mathrm{~d} \sigma \mathrm{d} t \\
&+2 \iint_{\Sigma} \frac{\partial \tilde{\psi}}{\partial n} \tilde{\psi}_{t} \mathrm{~d} \sigma \mathrm{d} t+2 s^{2} \lambda \iint_{\Sigma} \tilde{\alpha}_{t} \frac{\partial \eta^{0}}{\partial n} \tilde{\xi}|\tilde{\psi}|^{2} \mathrm{~d} \sigma \mathrm{d} t \\
& \leq C\left(\iint_{Q} \mathrm{e}^{-2 s \tilde{\alpha}}|f|^{2} \mathrm{~d} x \mathrm{~d} t+s^{3} \lambda^{4} \iint_{\omega \times(0, T)} \tilde{\xi}^{3}|\tilde{\psi}|^{2} \mathrm{~d} x \mathrm{~d} t\right)
\end{aligned}
$$

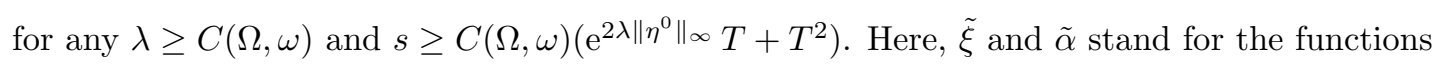

$$
\tilde{\xi}(x, t)=\frac{\mathrm{e}^{-\lambda \eta^{0}(x)}}{t(T-t)}, \quad \tilde{\alpha}(x, t)=\frac{\mathrm{e}^{2 \lambda\left\|\eta^{0}\right\|_{\infty}}-\mathrm{e}^{-\lambda \eta^{0}(x)}}{t(T-t)} .
$$

3 - Finally, we add the previous two inequalities and we come back to the original variable $\varphi$. This will give the desired inequality (15).

Step 1. Let us put $\psi=\mathrm{e}^{-s \alpha} q$. Since $-q_{t}-\Delta q=f$, we also have

$$
M_{1} \psi+M_{2} \psi=F
$$

where

$$
\begin{gathered}
M_{1} \psi=2 s \lambda^{2} \xi\left|\nabla \eta^{0}\right|^{2} \psi+2 s \lambda \xi \nabla \eta^{0} \cdot \nabla \psi-\psi_{t}, \\
M_{2} \psi=-s^{2} \lambda^{2} \xi^{2}\left|\nabla \eta^{0}\right|^{2} \psi-\Delta \psi-s \alpha_{t} \psi, \\
F=\mathrm{e}^{-s \alpha} f-s \lambda \xi \Delta \eta^{0} \psi+s \lambda^{2} \xi\left|\nabla \eta^{0}\right|^{2} \psi
\end{gathered}
$$

From (42), we have that

$$
\left\|M_{1} \psi\right\|_{L^{2}(Q)}^{2}+\left\|M_{2} \psi\right\|_{L^{2}(Q)}^{2}+2\left(M_{1} \psi, M_{2} \psi\right)_{L^{2}(Q)}=\|F\|_{L^{2}(Q)}^{2}
$$

The main idea is to expand the term $2\left(M_{1} \psi, M_{2} \psi\right)_{L^{2}(Q)}$ and use the particular structure of $\alpha$ and the fact that $s$ is large enough in order to obtain large positive terms in this scalar product. Denoting by $\left(M_{i} \psi\right)_{j}(1 \leq$ $i \leq 2,1 \leq j \leq 3)$ the $j$-th term in the above expression of $M_{i} \psi$, we find that

$$
\left(M_{1} \psi, M_{2} \psi\right)_{L^{2}(Q)}=\sum_{1 \leq i, j \leq 3}\left(\left(M_{1} \psi\right)_{i},\left(M_{2} \psi\right)_{j}\right)_{L^{2}(Q)}
$$

Let us compute each of these terms.

First, we have

$$
\left(\left(M_{1} \psi\right)_{1},\left(M_{2} \psi\right)_{1}\right)_{L^{2}(Q)}=-2 s^{3} \lambda^{4} \iint_{Q}\left|\nabla \eta^{0}\right|^{4} \xi^{3}|\psi|^{2} \mathrm{~d} x \mathrm{~d} t=A
$$


Then,

$$
\begin{aligned}
\left(\left(M_{1} \psi\right)_{2},\left(M_{2} \psi\right)_{1}\right)_{L^{2}(Q)}= & -2 s^{3} \lambda^{3} \iint_{Q}\left|\nabla \eta^{0}\right|^{2} \xi^{3}\left(\nabla \eta^{0} \cdot \nabla \psi\right) \psi \mathrm{d} x \mathrm{~d} t \\
= & 3 s^{3} \lambda^{4} \iint_{Q}\left|\nabla \eta^{0}\right|^{4} \xi^{3}|\psi|^{2} \mathrm{~d} x \mathrm{~d} t+s^{3} \lambda^{3} \iint_{Q} \Delta \eta^{0}\left|\nabla \eta^{0}\right|^{2} \xi^{3}|\psi|^{2} \mathrm{~d} x \mathrm{~d} t \\
& +2 s^{3} \lambda^{3} \iint_{Q} \partial_{i} \eta^{0} \partial_{i j} \eta^{0} \partial_{j} \eta^{0} \xi^{3}|\psi|^{2} \mathrm{~d} x \mathrm{~d} t \\
& -s^{3} \lambda^{3} \iint_{\Sigma}\left|\nabla \eta^{0}\right|^{2} \xi^{3} \frac{\partial \eta^{0}}{\partial n}|\psi|^{2} \mathrm{~d} \sigma \mathrm{d} t=B_{1}+B_{2}+B_{3}+B_{4} .
\end{aligned}
$$

We clearly have that $A+B_{1}$ is a positive term. As a consequence of the properties of $\eta^{0}$ (see (14)), we have

$$
s^{3} \lambda^{4} \iint_{Q}\left|\nabla \eta^{0}\right|^{4} \xi^{3}|\psi|^{2} \mathrm{~d} x \mathrm{~d} t \geq C s^{3} \lambda^{4} \iint_{Q} \xi^{3}|\psi|^{2} \mathrm{~d} x \mathrm{~d} t-C s^{3} \lambda^{4} \iint_{\omega^{\prime} \times(0, T)} \xi^{3}|\psi|^{2} \mathrm{~d} x \mathrm{~d} t
$$

for some $C=C(\Omega, \omega)$. The first of these last two integrals will stay in the left hand side and the second one will go to the right.

The boundary term $B_{4}$ will also stay in the left hand side, while $B_{2}$ and $B_{3}$ will be absorbed by simply taking $\lambda \geq C(\Omega, \omega)$.

We also have

$$
\begin{aligned}
\left(\left(M_{1} \psi\right)_{3},\left(M_{2} \psi\right)_{1}\right)_{L^{2}(Q)} & =s^{2} \lambda^{2} \iint_{Q}\left|\nabla \eta^{0}\right|^{2} \xi^{2} \psi_{t} \psi \mathrm{d} x \mathrm{~d} t \\
& =-s^{2} \lambda^{2} \iint_{Q}\left|\nabla \eta^{0}\right|^{2} \xi \xi_{t}|\psi|^{2} \mathrm{~d} x \mathrm{~d} t \leq C s^{2} \lambda^{2} T \iint_{Q} \xi^{3}|\psi|^{2} \mathrm{~d} x \mathrm{~d} t
\end{aligned}
$$

which is also absorbed by taking $\lambda \geq 1$ and $s \geq C(\Omega, \omega) T$.

Consequently, we obtain

$$
\begin{aligned}
\left(M_{1} \psi,\left(M_{2} \psi\right)_{1}\right)_{L^{2}(Q)}= & \left(\left(M_{1} \psi\right)_{1}+\left(M_{1} \psi\right)_{2}+\left(M_{1} \psi\right)_{3},\left(M_{2} \psi\right)_{1}\right)_{L^{2}(Q)} \\
\geq & C s^{3} \lambda^{4} \iint_{Q} \xi^{3}|\psi|^{2} \mathrm{~d} x \mathrm{~d} t-s^{3} \lambda^{3} \iint_{\Sigma}\left|\nabla \eta^{0}\right|^{2} \xi^{3} \frac{\partial \eta^{0}}{\partial n}|\psi|^{2} \mathrm{~d} \sigma \mathrm{d} t \\
& -C s^{3} \lambda^{4} \iint_{\omega^{\prime} \times(0, T)} \xi^{3}|\psi|^{2} \mathrm{~d} x \mathrm{~d} t
\end{aligned}
$$

for any $\lambda \geq C(\Omega, \omega)$ and $s \geq C(\Omega, \omega) T$.

On the other hand, we have

$$
\begin{aligned}
\left(\left(M_{1} \psi\right)_{1},\left(M_{2} \psi\right)_{2}\right)_{L^{2}(Q)}= & -2 s \lambda^{2} \iint_{Q}\left|\nabla \eta^{0}\right|^{2} \xi \Delta \psi \psi \mathrm{d} x \mathrm{~d} t \\
= & -2 s \lambda^{2} \iint_{\Sigma}\left|\nabla \eta^{0}\right|^{2} \xi \frac{\partial \psi}{\partial n} \psi \mathrm{d} \sigma \mathrm{d} t+2 s \lambda^{2} \iint_{Q}\left|\nabla \eta^{0}\right|^{2} \xi|\nabla \psi|^{2} \mathrm{~d} x \mathrm{~d} t \\
& +4 s \lambda^{2} \iint_{Q} \partial_{i} \eta^{0} \partial_{i j} \eta^{0} \xi \partial_{j} \psi \psi \mathrm{d} x \mathrm{~d} t+s \lambda^{3} \iint_{Q}\left|\nabla \eta^{0}\right|^{2} \xi \nabla \eta^{0} \cdot \nabla|\psi|^{2} \mathrm{~d} x \mathrm{~d} t \\
= & C_{1}+C_{2}+C_{3}+C_{4} .
\end{aligned}
$$


We will keep $C_{1}$ and $C_{2}$ in the left hand side. For $C_{3}$ and $C_{4}$, we have

$$
C_{3} \leq C s \lambda^{4} \iint_{Q} \xi|\psi|^{2} \mathrm{~d} x \mathrm{~d} t+C s \iint_{Q} \xi|\nabla \psi|^{2} \mathrm{~d} x \mathrm{~d} t
$$

and

$$
C_{4} \leq C s^{2} \lambda^{4} \iint_{Q} \xi^{2}|\psi|^{2} \mathrm{~d} x \mathrm{~d} t+C \lambda^{2} \iint_{Q}|\nabla \psi|^{2} \mathrm{~d} x \mathrm{~d} t .
$$

Therefore, by taking $s \geq C T^{2}$, we find that

$$
\begin{aligned}
C_{1}+C_{2}+C_{3} & +C_{4} \geq-2 s \lambda^{2} \iint_{\Sigma}\left|\nabla \eta^{0}\right|^{2} \xi \frac{\partial \psi}{\partial n} \psi \mathrm{d} \sigma \mathrm{d} t \\
& +2 s \lambda^{2} \iint_{Q}\left|\nabla \eta^{0}\right|^{2} \xi|\nabla \psi|^{2} \mathrm{~d} x \mathrm{~d} t-C s^{2} \lambda^{4} \iint_{Q} \xi^{2}|\psi|^{2} \mathrm{~d} x \mathrm{~d} t-C \iint_{Q}\left(s \xi+\lambda^{2}\right)|\nabla \psi|^{2} \mathrm{~d} x \mathrm{~d} t
\end{aligned}
$$

We also have

$$
\begin{aligned}
\left(\left(M_{1} \psi\right)_{2},\left(M_{2} \psi\right)_{2}\right)_{L^{2}(Q)}= & -2 s \lambda \iint_{Q} \xi\left(\nabla \eta^{0} \cdot \nabla \psi\right) \Delta \psi \mathrm{d} x \mathrm{~d} t \\
= & -2 s \lambda \iint_{\Sigma} \frac{\partial \eta^{0}}{\partial n} \xi\left|\frac{\partial \psi}{\partial n}\right|^{2} \mathrm{~d} \sigma \mathrm{d} t+2 s \lambda \iint_{Q} \partial_{i j} \eta^{0} \xi \partial_{i} \psi \partial_{j} \psi \mathrm{d} x \mathrm{~d} t \\
& +2 s \lambda^{2} \iint_{Q} \xi\left|\nabla \eta^{0} \cdot \nabla \psi\right|^{2} \mathrm{~d} x \mathrm{~d} t+s \lambda \iint_{Q} \xi \nabla \eta^{0} \cdot \nabla|\nabla \psi|^{2} \mathrm{~d} x \mathrm{~d} t \\
= & D_{1}+D_{2}+D_{3}+D_{4} .
\end{aligned}
$$

As before, we will keep the boundary integral $D_{1}$ in the left hand side. Also,

$$
D_{2} \leq C s \lambda \iint_{Q} \xi|\nabla \psi|^{2} \mathrm{~d} x \mathrm{~d} t .
$$

Moreover, $D_{3} \geq 0$. After some additional computations, we also see that

$$
\begin{aligned}
D_{4}= & s \lambda \iint_{Q} \xi \nabla \eta^{0} \cdot \nabla|\nabla \psi|^{2} \mathrm{~d} x \mathrm{~d} t=s \lambda \iint_{\Sigma} \xi \frac{\partial \eta^{0}}{\partial n}|\nabla \psi|^{2} \mathrm{~d} \sigma \mathrm{d} t \\
& -s \lambda^{2} \iint_{Q}\left|\nabla \eta^{0}\right|^{2} \xi|\nabla \psi|^{2} \mathrm{~d} x \mathrm{~d} t-s \lambda \iint_{Q} \Delta \eta^{0} \xi|\nabla \psi|^{2} \mathrm{~d} x \mathrm{~d} t \\
= & D_{41}+D_{42}+D_{43} .
\end{aligned}
$$

Now, we keep once more $D_{41}$ in the left and we notice that $D_{43}$ can be bounded in the same form as $D_{2}$.

Consequently,

$$
\begin{aligned}
D_{1}+D_{2}+D_{3}+D_{4} & \geq-2 s \lambda \iint_{\Sigma} \frac{\partial \eta^{0}}{\partial n} \xi\left|\frac{\partial \psi}{\partial n}\right|^{2} \mathrm{~d} \sigma \mathrm{d} t \\
& +s \lambda \iint_{\Sigma} \frac{\partial \eta^{0}}{\partial n} \xi|\nabla \psi|^{2} \mathrm{~d} \sigma \mathrm{d} t-s \lambda^{2} \iint_{Q}\left|\nabla \eta^{0}\right|^{2} \xi|\nabla \psi|^{2} \mathrm{~d} x \mathrm{~d} t-C s \lambda \iint_{Q} \xi|\nabla \psi|^{2} \mathrm{~d} x \mathrm{~d} t
\end{aligned}
$$


Additionally, we find that

$$
\begin{aligned}
\left(\left(M_{1} \psi\right)_{3},\left(M_{2} \psi\right)_{2}\right)_{L^{2}(Q)} & =\iint_{Q} \psi_{t} \Delta \psi \mathrm{d} x \mathrm{~d} t \\
& =\iint_{\Sigma} \frac{\partial \psi}{\partial n} \psi_{t} \mathrm{~d} \sigma \mathrm{d} t=E
\end{aligned}
$$

which will stay in the left hand side.

From (45)-(47), we deduce that

$$
\begin{aligned}
\left(M_{1} \psi,\left(M_{2} \psi\right)_{2}\right)_{L^{2}(Q)}= & \left(\left(M_{1} \psi\right)_{1}+\left(M_{1} \psi\right)_{2}+\left(M_{1} \psi\right)_{3},\left(M_{2} \psi\right)_{2}\right)_{L^{2}(Q)} \\
\geq & s \lambda^{2} \iint_{Q}\left|\nabla \eta^{0}\right|^{2} \xi|\nabla \psi|^{2} \mathrm{~d} x \mathrm{~d} t-2 s \lambda^{2} \iint_{\Sigma}\left|\nabla \eta^{0}\right|^{2} \xi \frac{\partial \psi}{\partial n} \psi \mathrm{d} \sigma \mathrm{d} t \\
& -2 s \lambda \iint_{\Sigma} \frac{\partial \eta^{0}}{\partial n} \xi\left|\frac{\partial \psi}{\partial n}\right|^{2} \mathrm{~d} \sigma \mathrm{d} t+s \lambda \iint_{\Sigma} \frac{\partial \eta^{0}}{\partial n} \xi|\nabla \psi|^{2} \mathrm{~d} x \mathrm{~d} t \\
& +\iint_{\Sigma} \frac{\partial \psi}{\partial n} \psi_{t} \mathrm{~d} \sigma \mathrm{d} t-C s^{2} \lambda^{4} \iint_{Q} \xi^{2}|\psi|^{2} \mathrm{~d} x \mathrm{~d} t-C \iint_{Q}\left(s \lambda \xi+\lambda^{2}\right)|\nabla \psi|^{2} \mathrm{~d} x \mathrm{~d} t
\end{aligned}
$$

for any $\lambda \geq 1$. Hence, we have the following for any $\lambda \geq C(\Omega, \omega)$ and any $s \geq C(\Omega, \omega) T^{2}$ :

$$
\begin{aligned}
& \left(M_{1} \psi,\left(M_{2} \psi\right)_{2}\right)_{L^{2}(Q)} \geq C s \lambda^{2} \iint_{Q} \xi|\nabla \psi|^{2} \mathrm{~d} x \mathrm{~d} t-2 s \lambda^{2} \iint_{\Sigma}\left|\nabla \eta^{0}\right|^{2} \xi \frac{\partial \psi}{\partial n} \psi \mathrm{d} \sigma \mathrm{d} t-2 s \lambda \iint_{\Sigma} \frac{\partial \eta^{0}}{\partial n} \xi\left|\frac{\partial \psi}{\partial n}\right|^{2} \mathrm{~d} \sigma \mathrm{d} t \\
& \quad+s \lambda \iint_{\Sigma} \frac{\partial \eta^{0}}{\partial n} \xi|\nabla \psi|^{2} \mathrm{~d} x \mathrm{~d} t+\iint_{\Sigma} \frac{\partial \psi}{\partial n} \psi_{t} \mathrm{~d} \sigma \mathrm{d} t-C s^{2} \lambda^{4} \iint_{Q} \xi^{2}|\psi|^{2} \mathrm{~d} x \mathrm{~d} t-C s \lambda^{2} \iint_{\omega^{\prime} \times(0, T)} \xi|\nabla \psi|^{2} \mathrm{~d} x \mathrm{~d} t .
\end{aligned}
$$

Let us now consider the scalar product

$$
\begin{aligned}
\left(\left(M_{1} \psi\right)_{1},\left(M_{2} \psi\right)_{3}\right)_{L^{2}(Q)} & =-2 s^{2} \lambda^{2} \iint_{Q}\left|\nabla \eta^{0}\right|^{2} \alpha_{t} \xi|\psi|^{2} \mathrm{~d} x \mathrm{~d} t \\
& \leq C(\Omega, \omega) \mathrm{e}^{2 \lambda\left\|\eta^{0}\right\|_{\infty}} s^{2} \lambda^{2} T \iint_{Q} \xi^{3}|\psi|^{2} \mathrm{~d} x \mathrm{~d} t
\end{aligned}
$$

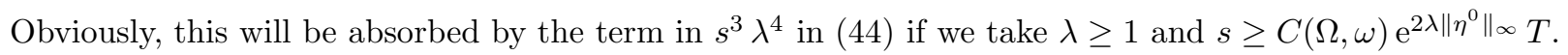

Furthermore,

$$
\begin{aligned}
\left(\left(M_{1} \psi\right)_{2},\left(M_{2} \psi\right)_{3}\right)_{L^{2}(Q)}= & -2 s^{2} \lambda \iint_{Q} \alpha_{t} \xi\left(\nabla \eta^{0} \cdot \nabla \psi\right) \psi \mathrm{d} x \mathrm{~d} t \\
= & -s^{2} \lambda \iint_{\Sigma} \alpha_{t} \frac{\partial \eta^{0}}{\partial n} \xi|\psi|^{2} \mathrm{~d} \sigma \mathrm{d} t+s^{2} \lambda^{2} \iint_{Q} \alpha_{t}\left|\nabla \eta^{0}\right|^{2} \xi|\psi|^{2} \mathrm{~d} x \mathrm{~d} t \\
& +s^{2} \lambda \iint_{Q} \nabla \alpha_{t} \cdot \nabla \eta^{0} \xi|\psi|^{2} \mathrm{~d} x \mathrm{~d} t+s^{2} \lambda \iint_{Q} \alpha_{t} \Delta \eta^{0} \xi|\psi|^{2} \mathrm{~d} x \mathrm{~d} t .
\end{aligned}
$$

With $\lambda \geq 1$, the last three terms in the left hand side can be bounded by

$$
C(\Omega, \omega) \mathrm{e}^{2 \lambda\left\|\eta^{0}\right\|_{\infty}} s^{2} \lambda^{2} T \iint_{Q} \xi^{3}|\psi|^{2} \mathrm{~d} x \mathrm{~d} t .
$$


Thus, we have

$$
\left(\left(M_{1} \psi\right)_{2},\left(M_{2} \psi\right)_{3}\right)_{L^{2}(Q)} \geq-s^{2} \lambda \iint_{\Sigma} \alpha_{t} \frac{\partial \eta^{0}}{\partial n} \xi|\psi|^{2} \mathrm{~d} x \mathrm{~d} t-C \mathrm{e}^{2 \lambda \| \eta^{0}} \| \infty s^{2} \lambda^{2} T \iint_{Q} \xi^{3}|\psi|^{2} \mathrm{~d} x \mathrm{~d} t .
$$

Finally, we have

$$
\left(\left(M_{1} \psi\right)_{3},\left(M_{2} \psi\right)_{3}\right)_{L^{2}(Q)}=s \iint_{Q} \alpha_{t} \psi_{t} \psi \mathrm{d} x \mathrm{~d} t \leq C \mathrm{e}^{2 \lambda\left\|\eta^{0}\right\|_{\infty}} s T^{2} \iint_{Q} \xi^{3}|\psi|^{2} \mathrm{~d} x \mathrm{~d} t,
$$

since

$$
\alpha_{t t} \leq C \mathrm{e}^{2 \lambda\left\|\eta^{0}\right\|_{\infty}} \xi^{2}\left(1+T^{2} \xi\right) \leq C \mathrm{e}^{2 \lambda\left\|\eta^{0}\right\|_{\infty}} T^{2} \xi^{3} .
$$

From (49)-(51), we deduce that, for $\lambda \geq C(\Omega, \omega)$ and $s \geq C(\Omega, \omega) \mathrm{e}^{2 \lambda \| \eta^{0}} \|_{\infty} T$, one has

$$
\begin{aligned}
\left(M_{1} \psi,\left(M_{2} \psi\right)_{3}\right)_{L^{2}(Q)} & =\left(\left(M_{1} \psi\right)_{1}+\left(M_{1} \psi\right)_{2}+\left(M_{1} \psi\right)_{3},\left(M_{2} \psi\right)_{3}\right)_{L^{2}(Q)} \\
& \geq G-C s^{3} \lambda^{2} \iint_{Q} \xi^{3}|\psi|^{2} \mathrm{~d} x \mathrm{~d} t
\end{aligned}
$$

where

$$
G=-s^{2} \lambda \iint_{\Sigma} \alpha_{t} \frac{\partial \eta^{0}}{\partial n} \xi|\psi|^{2} \mathrm{~d} \sigma \mathrm{d} t
$$

Taking into account (44), (48) and (52), we obtain

$$
\begin{aligned}
\left(M_{1} \psi, M_{2} \psi\right)_{L^{2}(Q) \geq} \geq & C \iint_{Q}\left(s \lambda^{2} \xi|\nabla \psi|^{2}+s^{3} \lambda^{4} \xi^{3}|\psi|^{2}\right) \mathrm{d} x \mathrm{~d} t \\
& -s^{3} \lambda^{3} \iint_{\Sigma}\left|\nabla \eta^{0}\right|^{2} \xi^{3} \frac{\partial \eta^{0}}{\partial n}|\psi|^{2} \mathrm{~d} \sigma \mathrm{d} t-2 s \lambda^{2} \iint_{\Sigma}\left|\nabla \eta^{0}\right|^{2} \xi \frac{\partial \psi}{\partial n} \psi \mathrm{d} \sigma \mathrm{d} t \\
& -2 s \lambda \iint_{\Sigma} \frac{\partial \eta^{0}}{\partial n} \xi\left|\frac{\partial \psi}{\partial n}\right|^{2} \mathrm{~d} \sigma \mathrm{d} t+s \lambda \iint_{\Sigma} \frac{\partial \eta^{0}}{\partial n} \xi|\nabla \psi|^{2} \mathrm{~d} x \mathrm{~d} t \\
& +\iint_{\Sigma} \frac{\partial \psi}{\partial n} \psi_{t} \mathrm{~d} \sigma \mathrm{d} t-s^{2} \lambda \iint_{\Sigma} \alpha_{t} \frac{\partial \eta^{0}}{\partial n} \xi|\psi|^{2} \mathrm{~d} \sigma \mathrm{d} t \\
& -C \iint_{\omega^{\prime} \times(0, T)}\left(s \lambda^{2} \xi|\nabla \psi|^{2}+s^{3} \lambda^{4} \xi^{3}|\psi|^{2}\right) \mathrm{d} x \mathrm{~d} t
\end{aligned}
$$

for any $\lambda \geq C(\Omega, \omega)$ and $s \geq C(\Omega, \omega)\left(\mathrm{e}^{2 \lambda\left\|\eta^{0}\right\|_{\infty}} T+T^{2}\right)$. Using (43), this gives

$$
\begin{aligned}
&\left\|M_{1} \psi\right\|_{L^{2}(Q)}^{2}+\left\|M_{2} \psi\right\|_{L^{2}(Q)}^{2}+ \iint_{Q}\left(s \lambda^{2} \xi|\nabla \psi|^{2}+s^{3} \lambda^{4} \xi^{3}|\psi|^{2}\right) \mathrm{d} x \mathrm{~d} t \\
&+2\left(B_{4}+C_{1}+D_{1}+D_{41}+E+G\right) \leq C\left(\|F\|_{L^{2}(Q)}^{2}\right. \\
&\left.\left.+s \lambda^{2} \iint_{\omega^{\prime} \times(0, T)} \xi|\nabla \psi|^{2} \mathrm{~d} x \mathrm{~d} t+s^{3} \lambda^{4} \iint_{\omega^{\prime} \times(0, T)} \xi^{3}|\psi|^{2}\right) \mathrm{~d} x \mathrm{~d} t\right) \\
& \leq C\left(\iint_{Q} \mathrm{e}^{-2 s \alpha}|f|^{2} \mathrm{~d} x \mathrm{~d} t+s^{2} \lambda^{4} \iint_{Q} \xi^{2}|\psi|^{2} \mathrm{~d} x \mathrm{~d} t\right. \\
&\left.\left.+s \lambda^{2} \iint_{\omega^{\prime} \times(0, T)} \xi|\nabla \psi|^{2} \mathrm{~d} x \mathrm{~d} t+s^{3} \lambda^{4} \iint_{\omega^{\prime} \times(0, T)} \xi^{3}|\psi|^{2}\right) \mathrm{~d} x \mathrm{~d} t\right) .
\end{aligned}
$$


Thus, we also have

$$
\begin{aligned}
\left\|M_{1} \psi\right\|_{L^{2}(Q)}^{2}+\left\|M_{2} \psi\right\|_{L^{2}(Q)}^{2} & +\iint_{Q}\left(s \lambda^{2} \xi|\nabla \psi|^{2}+s^{3} \lambda^{4} \xi^{3}|\psi|^{2}\right) \mathrm{d} x \mathrm{~d} t \\
+2\left(B_{4}+C_{1}+D_{1}+D_{41}+E+G\right) & \leq C\left(\iint_{Q} \mathrm{e}^{-2 s \alpha}|f|^{2} \mathrm{~d} x \mathrm{~d} t\right. \\
& \left.\left.+s \lambda^{2} \iint_{\omega^{\prime} \times(0, T)} \xi|\nabla \psi|^{2} \mathrm{~d} x \mathrm{~d} t+s^{3} \lambda^{4} \iint_{\omega^{\prime} \times(0, T)} \xi^{3}|\psi|^{2}\right) \mathrm{~d} x \mathrm{~d} t\right)
\end{aligned}
$$

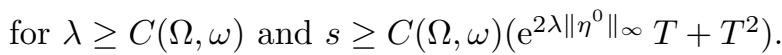

The next step is to try to add integrals of $|\Delta \psi|^{2}$ and $\left|\psi_{t}\right|^{2}$ to the left hand side of (53). This can be made using the expressions of $M_{i} \psi(i=1,2)$. Indeed, we have

$$
s^{-1} \iint_{Q} \xi^{-1}\left|\psi_{t}\right|^{2} \mathrm{~d} x \mathrm{~d} t \leq C\left(s \lambda^{2} \iint_{Q} \xi|\nabla \psi|^{2} \mathrm{~d} x \mathrm{~d} t+s \lambda^{4} \iint_{Q} \xi|\psi|^{2} \mathrm{~d} x \mathrm{~d} t+\left\|M_{1} \psi\right\|_{L^{2}(Q)}^{2}\right)
$$

and

$$
s^{-1} \iint_{Q} \xi^{-1}|\Delta \psi|^{2} \mathrm{~d} x \mathrm{~d} t \leq C\left(s^{3} \lambda^{4} \iint_{Q} \xi^{3}|\psi|^{2} \mathrm{~d} x \mathrm{~d} t+s T^{2} \mathrm{e}^{\left.4 \lambda\left\|\eta^{0}\right\|\left\|_{\infty} \iint_{Q} \xi^{3}|\psi|^{2} \mathrm{~d} x \mathrm{~d} t+\right\| M_{2} \psi \|_{L^{2}(Q)}^{2}\right)}\right.
$$

for $s \geq C T^{2}$. Accordingly, we deduce from (53) that

$$
\begin{aligned}
& \iint_{Q}\left((s \xi)^{-1}\left(\left|\psi_{t}\right|^{2}+|\Delta \psi|^{2}\right)\right.\left.+s \lambda^{2} \xi|\nabla \psi|^{2}+s^{3} \lambda^{4} \xi^{3}|\psi|^{2}\right) \mathrm{d} x \mathrm{~d} t \\
&+2\left(B_{4}+C_{1}+D_{1}+D_{41}+E+G\right) \leq C\left(\iint_{Q} \mathrm{e}^{-2 s \alpha}|f|^{2} \mathrm{~d} x \mathrm{~d} t\right. \\
&\left.\left.+s \lambda^{2} \iint_{\omega^{\prime} \times(0, T)} \xi|\nabla \psi|^{2} \mathrm{~d} x \mathrm{~d} t+s^{3} \lambda^{4} \iint_{\omega^{\prime} \times(0, T)} \xi^{3}|\psi|^{2}\right) \mathrm{~d} x \mathrm{~d} t\right)
\end{aligned}
$$

for $\lambda \geq C(\Omega, \omega)$ and $s \geq C(\Omega, \omega)\left(\mathrm{e}^{2 \lambda\left\|\eta^{0}\right\|_{\infty}} T+T^{2}\right)$.

We are now ready to eliminate the second integral in the right hand side. To this end, let us introduce a function $\theta=\theta(x)$, with

$$
\theta \in C_{c}^{2}(\omega), \quad \theta \equiv 1 \text { in } \omega^{\prime}, \quad 0 \leq \theta \leq 1
$$

and let us make some computations:

$$
\begin{aligned}
s \lambda^{2} \iint_{\omega^{\prime} \times(0, T)} \xi|\nabla \psi|^{2} \mathrm{~d} x \mathrm{~d} t \leq & s \lambda^{2} \iint_{\omega \times(0, T)} \theta \xi|\nabla \psi|^{2} \mathrm{~d} x \mathrm{~d} t \\
= & -s \lambda^{2} \iint_{\omega \times(0, T)} \theta \xi \Delta \psi \psi \mathrm{d} x \mathrm{~d} t-s \lambda^{2} \iint_{\omega \times(0, T)} \xi(\nabla \theta \cdot \nabla \psi) \psi \mathrm{d} x \mathrm{~d} t \\
& -s \lambda^{3} \iint_{\omega \times(0, T)} \theta \xi\left(\nabla \eta^{0} \cdot \nabla \psi\right) \psi \mathrm{d} x \mathrm{~d} t \leq \varepsilon s^{-1} \iint_{\omega \times(0, T)} \xi^{-1}|\Delta \psi|^{2} \mathrm{~d} x \mathrm{~d} t \\
& +C\left(s^{3} \lambda^{4} \iint_{\omega \times(0, T)} \xi^{3}|\psi|^{2} \mathrm{~d} x \mathrm{~d} t+s \lambda^{4} \iint_{\omega \times(0, T)} \xi|\psi|^{2} \mathrm{~d} x \mathrm{~d} t\right)
\end{aligned}
$$


where we have used that $\lambda \geq 1$. In view of this estimate, we deduce that the integral on $|\nabla \psi|^{2}$ of the right hand side of (54) can be suppressed if the last integral is performed in the slightly greater set $\omega \times(0, T)$. From $(54)$ and this remark, we deduce (40).

Step 2. The proof of (41) is very similar to the proof of (40). We will only sketch the main points.

We start from the identity

$$
M_{1} \tilde{\psi}+M_{2} \tilde{\psi}=\tilde{F}
$$

where

$$
\begin{gathered}
\tilde{M}_{1} \tilde{\psi}=2 s \lambda^{2}\left|\nabla \eta^{0}\right|^{2} \tilde{\xi} \tilde{\psi}-2 s \lambda \tilde{\xi} \nabla \eta^{0} \cdot \nabla \tilde{\psi}-\tilde{\psi}_{t}, \\
\tilde{M}_{2} \tilde{\psi}=-s^{2} \lambda^{2}\left|\nabla \eta^{0}\right|^{2} \tilde{\xi}^{2} \tilde{\psi}-\Delta \tilde{\psi}-s \tilde{\alpha} t \tilde{\psi} \\
\tilde{F}=\mathrm{e}^{-s \tilde{\alpha}} f+s \lambda \tilde{\xi} \Delta \eta^{0} \tilde{\psi}+s \lambda^{2}\left|\nabla \eta^{0}\right|^{2} \tilde{\xi} \tilde{\psi} .
\end{gathered}
$$

We then have

$$
\left\|\tilde{M}_{1} \tilde{\psi}\right\|_{L^{2}(Q)}^{2}+\left\|\tilde{M}_{2} \tilde{\psi}\right\|_{L^{2}(Q)}^{2}+2\left(\tilde{M}_{1} \tilde{\psi}, \tilde{M}_{2} \tilde{\psi}\right)_{L^{2}(Q)}=\|\tilde{F}\|_{L^{2}(Q)}^{2} .
$$

After a lengthy computation, we find that

$$
\begin{aligned}
\left(\tilde{M}_{1} \psi, \tilde{M}_{2} \psi\right)_{L^{2}(Q) \geq} \geq & C \iint_{Q}\left(s \lambda^{2} \tilde{\xi}|\nabla \tilde{\psi}|^{2}+s^{3} \lambda^{4} \tilde{\xi}^{3}|\tilde{\psi}|^{2}\right) \mathrm{d} x \mathrm{~d} t \\
& +2\left(\tilde{B}_{4}+\tilde{C}_{1}+\tilde{D}_{1}+\tilde{D}_{41}+\tilde{E}+\tilde{G}\right)-C \iint_{\omega^{\prime} \times(0, T)}\left(s \lambda^{2} \tilde{\xi}|\nabla \tilde{\psi}|^{2}+s^{3} \lambda^{4} \tilde{\xi}^{3}|\tilde{\psi}|^{2}\right) \mathrm{d} x \mathrm{~d} t
\end{aligned}
$$

for any $\lambda \geq C(\Omega, \omega)$ and $s \geq C(\Omega, \omega)\left(\mathrm{e}^{2 \lambda\left\|\eta^{0}\right\|_{\infty}} T+T^{2}\right)$, where

$$
\begin{gathered}
\tilde{B}_{4}=s^{3} \lambda^{3} \iint_{\Sigma}\left|\nabla \eta^{0}\right|^{2} \tilde{\xi}^{3} \frac{\partial \eta^{0}}{\partial n}|\tilde{\psi}|^{2} \mathrm{~d} \sigma \mathrm{d} t \\
\tilde{C}_{1}=-2 s \lambda^{2} \iint_{\Sigma}\left|\nabla \eta^{0}\right|^{2} \tilde{\xi} \frac{\partial \tilde{\psi}}{\partial n} \tilde{\psi} \mathrm{d} \sigma \mathrm{d} t \\
\tilde{D}_{1}=2 s \lambda \iint_{\Sigma} \frac{\partial \eta^{0}}{\partial n} \tilde{\xi}\left|\frac{\partial \tilde{\psi}}{\partial n}\right|^{2} \mathrm{~d} \sigma \mathrm{d} t, \quad \tilde{D}_{41}=-s \lambda \iint_{\Sigma} \frac{\partial \eta^{0}}{\partial n} \tilde{\xi}|\nabla \tilde{\psi}|^{2} \mathrm{~d} x \mathrm{~d} t \\
\tilde{E}=\iint_{\Sigma} \frac{\partial \tilde{\psi}}{\partial n} \tilde{\psi}_{t} \mathrm{~d} \sigma \mathrm{d} t, \quad \tilde{G}=s^{2} \lambda \iint_{\Sigma} \tilde{\alpha}_{t} \frac{\partial \eta^{0}}{\partial n} \tilde{\xi}|\tilde{\psi}|^{2} \mathrm{~d} \sigma \mathrm{d} t .
\end{gathered}
$$

This, together with (55), gives

$$
\begin{aligned}
\left\|M_{1} \tilde{\psi}\right\|_{L^{2}(Q)}^{2}+\left\|M_{2} \tilde{\psi}\right\|_{L^{2}(Q)}^{2}+ & \iint_{Q}\left(s \lambda^{2} \tilde{\xi}|\nabla \tilde{\psi}|^{2}+s^{3} \lambda^{4} \tilde{\xi}^{3}|\tilde{\psi}|^{2}\right) \mathrm{d} x \mathrm{~d} t \\
& +2\left(\tilde{B}_{4}+\tilde{C}_{1}+\tilde{D}_{1}+\tilde{D}_{41}+\tilde{E}+\tilde{G}\right) \leq C\left(\|\tilde{F}\|_{L^{2}(Q)}^{2}\right. \\
& \left.\left.\quad+s \lambda^{2} \iint_{\omega^{\prime} \times(0, T)} \tilde{\xi}|\nabla \tilde{\psi}|^{2} \mathrm{~d} x \mathrm{~d} t+s^{3} \lambda^{4} \iint_{\omega^{\prime} \times(0, T)} \tilde{\xi}^{3}|\tilde{\psi}|^{2}\right) \mathrm{~d} x \mathrm{~d} t\right) .
\end{aligned}
$$

With similar arguments to those in the first step, we can now assume that, in $(56),\|\tilde{F}\|_{L^{2}(Q)}^{2}$ is replaced by

$$
\iint_{Q} \mathrm{e}^{-2 s \tilde{\alpha}}|f|^{2} \mathrm{~d} x \mathrm{~d} t
$$


and $\left\|\tilde{M}_{1} \tilde{\psi}\right\|_{L^{2}(Q)}^{2}+\left\|\tilde{M}_{2} \tilde{\psi}\right\|_{L^{2}(Q)}^{2}$ is replaced by

$$
\iint_{Q} s^{-1} \xi^{-1}\left(\left|\tilde{\psi}_{t}\right|^{2}+|\Delta \tilde{\psi}|^{2}\right) \mathrm{d} x \mathrm{~d} t .
$$

Finally, for $\lambda \geq C(\Omega, \omega)$ and $s \geq C(\Omega, \omega) T^{2}$ large enough, we can replace the integrals of $|\nabla \tilde{\psi}|^{2}$ and $|\tilde{\psi}|^{2}$ in the right hand side by

$$
s^{3} \lambda^{4} \iint_{\omega \times(0, T)} \tilde{\xi}^{3}|\tilde{\psi}|^{2} \mathrm{~d} x \mathrm{~d} t .
$$

This yields the estimate (41).

Step 3. Now, let us add the inequalities (40) and (41) and let us check that all the integrals on $\Sigma$ can be simplified, so that there will only remain integrals in $Q$.

Since $\eta^{0}=0$ on $\partial \Omega$, we have

$$
\xi=\tilde{\xi}, \quad \alpha=\tilde{\alpha} \quad \text { and } \quad \psi=\tilde{\psi} \quad \text { on } \Sigma .
$$

Consequently, $B_{4}+\tilde{B}_{4}=0$ and $G+\tilde{G}=0$.

Let us see that

$$
\frac{\partial \tilde{\psi}}{\partial n} \equiv-\frac{\partial \psi}{\partial n} \text { on } \Sigma \text {. }
$$

From the definitions of $\psi$ and $\tilde{\psi}$, we have

$$
\partial_{i} \psi=\mathrm{e}^{-s \alpha}\left(\partial_{i} q+s \lambda \partial_{i} \eta^{0} \xi q\right), \partial_{i} \tilde{\psi}=\mathrm{e}^{-s \tilde{\alpha}}\left(\partial_{i} q-s \lambda \partial_{i} \eta^{0} \tilde{\xi} q\right)
$$

whence

and we certainly have (58).

$$
\frac{\partial \psi}{\partial n}=s \lambda \frac{\partial \eta^{0}}{\partial n} \xi \mathrm{e}^{-s \alpha} q, \quad \frac{\partial \tilde{\psi}}{\partial n}=-s \lambda \frac{\partial \eta^{0}}{\partial n} \tilde{\xi} \mathrm{e}^{-s \tilde{\alpha}} q \quad \text { on } \quad \Sigma
$$

We deduce from (57) and (58) that $C_{1}+\tilde{C}_{1}=0, D_{1}+\tilde{D}_{1}=0$ and $E+\tilde{E}=0$.

On the other hand, since $\varphi$ satisfies a zero Neumann condition and $\eta^{0}=0$ on $\partial \Omega$, we also have

$$
|\nabla \psi|^{2}=|\nabla \tilde{\psi}|^{2} \quad \text { on } \Sigma
$$

whence $D_{41}+\tilde{D}_{41}=0$.

With all this, we obtain

$$
\begin{aligned}
s^{-1} \iint_{Q}\left(\xi ^ { - 1 } \left(\left|\psi_{t}\right|^{2}\right.\right. & \left.\left.+|\Delta \psi|^{2}\right)+\tilde{\xi}^{-1}\left(\left|\tilde{\psi}_{t}\right|^{2}+|\Delta \tilde{\psi}|^{2}\right)\right) \mathrm{d} x \mathrm{~d} t \\
& +s \lambda^{2} \iint_{Q}\left(\xi|\nabla \psi|^{2}+\tilde{\xi}|\nabla \tilde{\psi}|^{2}\right) \mathrm{d} x \mathrm{~d} t+s^{3} \lambda^{4} \iint_{Q}\left(\xi^{3}|\psi|^{2}+\tilde{\xi}^{3}|\tilde{\psi}|^{2}\right) \mathrm{d} x \mathrm{~d} t \\
& \leq C\left(s^{3} \lambda^{4} \iint_{\omega \times(0, T)}\left(\xi^{3}|\psi|^{2}+\tilde{\xi}^{3}|\tilde{\psi}|^{2}\right) \mathrm{d} x \mathrm{~d} t+\iint_{Q}\left(\mathrm{e}^{-2 s \alpha}+\mathrm{e}^{-2 s \tilde{\alpha}}\right)|f|^{2} \mathrm{~d} x \mathrm{~d} t\right)
\end{aligned}
$$

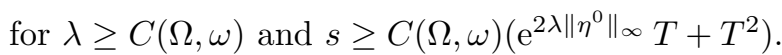

From the definitions of $\xi, \tilde{\xi}, \alpha$ and $\tilde{\alpha}$, we have

$$
\tilde{\xi} \leq \xi, \quad \mathrm{e}^{-2 s \tilde{\alpha}} \leq \mathrm{e}^{-2 s \alpha} \quad \text { in } Q,
$$


so (60) yields

$$
\begin{aligned}
\iint_{Q}\left((s \xi)^{-1}\left(\left|\psi_{t}\right|^{2}+|\Delta \psi|^{2}\right)+s \lambda^{2} \xi|\nabla \psi|^{2}\right. & \left.+s^{3} \lambda^{4} \xi^{3}|\psi|^{2}\right) \mathrm{d} x \mathrm{~d} t \\
& \leq C\left(\iint_{Q} \mathrm{e}^{-2 s \alpha}|f|^{2} \mathrm{~d} x \mathrm{~d} t+s^{3} \lambda^{4} \iint_{\omega \times(0, T)} \xi^{3}|\psi|^{2} \mathrm{~d} x \mathrm{~d} t\right)
\end{aligned}
$$

for any $\lambda \geq C(\Omega, \omega)$ and $s \geq C(\Omega, \omega)\left(\mathrm{e}^{2 \lambda\left\|\eta^{0}\right\|_{\infty}} T+T^{2}\right)$.

We finally turn back to $\varphi$. For the moment, we have

$$
\begin{aligned}
s^{-1} \iint_{Q} \xi^{-1}\left(\left|\psi_{t}\right|^{2}+|\Delta \psi|^{2}\right) \mathrm{d} x \mathrm{~d} t & +s \lambda^{2} \iint_{Q} \xi|\nabla \psi|^{2} \mathrm{~d} x \mathrm{~d} t+s^{3} \lambda^{4} \iint_{Q} \mathrm{e}^{-2 s \alpha} \xi^{3}|q|^{2} \mathrm{~d} x \mathrm{~d} t \\
& \leq C\left(\iint_{Q} \mathrm{e}^{-2 s \alpha}|f|^{2} \mathrm{~d} x \mathrm{~d} t+s^{3} \lambda^{4} \iint_{\omega \times(0, T)} \mathrm{e}^{-2 s \alpha} \xi^{3}|q|^{2} \mathrm{~d} x \mathrm{~d} t\right) .
\end{aligned}
$$

Using (59), we find that

$$
s \lambda^{2} \iint_{Q} \mathrm{e}^{-2 s \alpha} \xi|\nabla q|^{2} \mathrm{~d} x \mathrm{~d} t \leq C s \lambda^{2} \iint_{Q} \xi|\nabla \psi|^{2} \mathrm{~d} x \mathrm{~d} t+C s^{3} \lambda^{4} \iint_{Q} \mathrm{e}^{-2 s \alpha} \xi^{3}|q|^{2} \mathrm{~d} x \mathrm{~d} t .
$$

Accordingly, the previous integral of $|\nabla q|^{2}$ can be added to the left hand side of (62):

$$
\begin{array}{r}
s^{-1} \iint_{Q} \xi^{-1}\left|\psi_{t}\right|^{2} \mathrm{~d} x \mathrm{~d} t+s^{-1} \iint_{Q} \xi^{-1}|\Delta \psi|^{2} \mathrm{~d} x \mathrm{~d} t+s \lambda^{2} \iint_{Q} \xi|\nabla q|^{2} \mathrm{~d} x \mathrm{~d} t+s^{3} \lambda^{4} \iint_{Q} \mathrm{e}^{-2 s \alpha} \xi^{3}|q|^{2} \mathrm{~d} x \mathrm{~d} t \\
\leq C\left(s^{3} \lambda^{4} \iint_{\omega \times(0, T)} \mathrm{e}^{-2 s \alpha} \xi^{3}|q|^{2} \mathrm{~d} x \mathrm{~d} t+\iint_{Q} \mathrm{e}^{-2 s \alpha}|f|^{2} \mathrm{~d} x \mathrm{~d} t\right) .
\end{array}
$$

For $\Delta q$, we use the identity

$$
\Delta \psi=\mathrm{e}^{-s \alpha}\left(\Delta q+s \lambda \Delta \eta^{0} \xi q+s \lambda^{2}\left|\nabla \eta^{0}\right|^{2} \xi q+2 s \lambda \xi \nabla \eta^{0} \cdot \nabla q+s^{2} \lambda^{2}\left|\nabla \eta^{0}\right|^{2} \xi^{2} q\right)
$$

and we obtain

$$
\begin{aligned}
& s^{-1} \iint_{Q} \mathrm{e}^{-2 s \alpha} \xi^{-1}|\Delta q|^{2} \mathrm{~d} x \mathrm{~d} t \leq C\left(s^{-1} \iint_{Q} \xi^{-1}|\Delta \psi|^{2} \mathrm{~d} x \mathrm{~d} t\right. \\
&+s \lambda^{2} \iint_{Q} \mathrm{e}^{-2 s \alpha} \xi|q|^{2} \mathrm{~d} x \mathrm{~d} t+s \lambda^{4} \iint_{Q} \mathrm{e}^{-2 s \alpha} \xi|q|^{2} \mathrm{~d} x \mathrm{~d} t \\
&\left.\quad+s \lambda^{2} \iint_{Q} \mathrm{e}^{-2 s \alpha} \xi|\nabla q|^{2} \mathrm{~d} x \mathrm{~d} t+s^{3} \lambda^{4} \iint_{Q} \mathrm{e}^{-2 s \alpha} \xi^{3}|q|^{2} \mathrm{~d} x \mathrm{~d} t\right) .
\end{aligned}
$$

Finally, for $q_{t}$, we get

$$
s^{-1} \iint_{Q} \mathrm{e}^{-2 s \alpha} \xi^{-1}\left|q_{t}\right|^{2} \mathrm{~d} x \mathrm{~d} t \leq C(\Omega, \omega)\left(s^{-1} \iint_{Q} \xi^{-1}\left|\psi_{t}\right|^{2} \mathrm{~d} x \mathrm{~d} t+s \mathrm{e}^{4 \lambda\left\|\eta^{0}\right\|_{C(\bar{\Omega})}} T^{2} \iint_{Q} \mathrm{e}^{-2 s \alpha} \xi^{3}|q|^{2} \mathrm{~d} x \mathrm{~d} t\right),
$$

where we have used the identity

$$
q_{t}=\mathrm{e}^{s \alpha}\left(\psi_{t}+s \alpha_{t} \psi\right)
$$


Thus, taking $\lambda \geq 1$ and $s \geq C(\Omega, \omega)\left(\mathrm{e}^{2 \lambda\left\|\eta^{0}\right\|_{\infty}} T+T^{2}\right)$, we are able to introduce all terms of $I_{s, \lambda}(q)$ in the left hand side of (62). This yields (15) and concludes the proof of Lemma 1.

\section{REFERENCES}

[1] V. Barbu, Controllability of parabolic and Navier-Stokes equations. Sci. Math. Jpn 56 (2002) 143-211.

[2] A. Doubova, E. Fernández-Cara and M. González-Burgos, On the controllability of the heat equation with nonlinear boundary Fourier conditions. J. Diff. Equ. 196 (2004) 385-417.

[3] C. Fabre, J.P. Puel and E. Zuazua, Approximate controllability of the semilinear heat equation. Proc. Roy. Soc. Edinburgh 125A (1995) 31-61.

[4] E. Fernández-Cara and E. Zuazua, The cost of approximate controllability for heat equations: the linear case. Adv. Diff. Equ. 5 (2000) 465-514.

[5] A. Fursikov and O.Yu. Imanuvilov, Controllability of Evolution Equations. Lecture Notes no. 34, Seoul National University, Korea, 1996.

[6] O.Yu. Imanuvilov and M. Yamamoto, Carleman estimate for a parabolic equation in a Sobolev space of negative order and its applications, Dekker, New York. Lect. Notes Pure Appl. Math. 218 (2001).

[7] G. Lebeau and L. Robbiano, Contrôle exacte de l'equation de la chaleur (French). Comm. Partial Differ. Equat. 20 (1995) 335-356.

[8] D.L. Russell, A unified boundary controllability theory for hyperbolic and parabolic partial differential equations. Studies Appl. Math. 52 (1973) 189-211. 